\title{
$\gamma$ Cas stars: Normal Be stars with discs impacted by the wind of a helium-star companion?
}

\author{
N. Langer ${ }^{1,2}$, D. Baade ${ }^{3}$, J. Bodensteiner ${ }^{4}$, J. Greiner ${ }^{5}$, Th. Rivinius ${ }^{6}$, Ch. Martayan $^{6}$, and C. C. Borre ${ }^{7}$ \\ 1 Argelander Institut für Astronomie der Universität Bonn, Auf dem Hügel 71, 53121 Bonn, Germany \\ e-mail: nlanger@astro.uni-bonn.de \\ 2 Max-Planck-Institut für Radioastronomie, Auf dem Hügel 69, 53121 Bonn, Germany \\ ${ }^{3}$ European Organisation for Astronomical Research in the Southern Hemisphere (ESO), Karl-Schwarzschild-Str. 2, \\ 85748 Garching bei München, Germany \\ e-mail: dbaade@eso.org \\ 4 Instituut voor Sterrenkunde, KU Leuven, Celestijnenlaan 200D, Bus 2401, 3001 Leuven, Belgium \\ e-mail: julia.bodensteiner@kuleuven.be \\ 5 Max-Planck-Institut für Extraterrestrische Physik, Giessenbachstr. 1, 85748 Garching bei München, Germany \\ 6 European Organisation for Astronomical Research in the Southern Hemisphere (ESO), Casilla 19001, Santiago, Chile \\ 7 Stellar Astrophysics Centre, Aarhus University, Ny Munkegade 120, 8000 Aarhus C, Denmark
}

Received 19 September 2019 / Accepted 10 November 2019

\begin{abstract}
$\gamma$ Cas stars are a $\sim 1 \%$ minority among classical Be stars with hard $(\geq 5-10 \mathrm{keV})$, but only moderately strong continuous thermal $\mathrm{X}$-ray flux, and mostly very early-B spectral type. The X-ray flux has been suggested to originate from matter accelerated via magnetic disc-star interaction, by a rapidly rotating neutron star (NS) companion via the propeller effect, or by accretion onto a white dwarf (WD) companion. In view of the growing number of identified $\gamma$ Cas stars and the only imperfect matches between these suggestions and the observations, alternative models should be pursued. Two of the three best-observed $\gamma$ Cas stars, $\gamma$ Cas itself and $\pi$ Aqr, have a low-mass companion with low optical flux, whereas interferometry of BZ Cru is inconclusive. Binary-evolution models are examined for their ability to produce such systems. The $\mathrm{OB}+\mathrm{He}$-star stage of post-mass transfer binaries, which is otherwise observationally unaccounted, can potentially reproduce many observed properties of $\gamma$ Cas stars. The interaction of the fast wind of helium stars with the circumstellar disc and/or with the wind of Be stars may give rise to the production of hard X-rays. While not modelling this process, it is shown that the energy budget is favourable, and that the wind velocities may lead to hard X-rays, as observed in $\gamma$ Cas stars. Furthermore, the observed number of these objects appears to be consistent with the evolutionary models. Within the Be+He-star binary model, the Be stars in $\gamma$-Cas stars are conventional classical Be stars. They are encompassed by O-star+Wolf-Rayet systems towards higher mass, where no stable Be decretion discs exist, and by $\mathrm{Be}+\mathrm{sdO}$ systems at lower mass, where the sdO winds may be too weak to cause the $\gamma$ Cas phenomenon. In decreasing order of the helium-star mass, the descendants could be Be+black-hole, $\mathrm{Be}+\mathrm{NS}$, or $\mathrm{Be}+\mathrm{WD}$ binaries. The interaction between the helium-star wind and the disc may provide new diagnostics of the outer disc.
\end{abstract}

Key words. stars: emission-line, Be - binaries: general $-\mathrm{X}$-rays: stars - stars: individual: $\gamma \mathrm{Cas}-$ stars: individual: BZ Cru stars: individual: $\pi$ Aqr

\section{Introduction}

Many of the most rapidly rotating non-supergiant $\mathrm{B}$, late $\mathrm{O}$, and early A stars exhibit $\mathrm{H} \alpha$ line emission (Zorec \& Briot 1997; Yudin 2001). Typically, the emission lines form in a Keplerian disc and the central stars rotate at very roughly $80 \%$ of the critical velocity (Meilland et al. 2012). Stars with these properties are commonly called (classical) Be stars which were broadly reviewed by Rivinius et al. (2013). The Be Star Spectra database (BeSS, Neiner et al. 2011) lists nearly 250 Be stars with $v \leq 6.5$ mag.

Struve (1931) suggested that the discs of Be stars are the result of stellar rotational instability. On the one hand, the paucity of Be stars observed to rotate critically (Rivinius et al. 2013) appears to invalidate this simple hypothesis as a general property of Be stars. On the other hand, during the course of stellar evolution, core contraction and envelope expansion would combine to a net outward angular-momentum transport which, given a sufficient initial supply (as found by Martayan et al. 2007), could eventually lead to critical rotation (Granada et al. 2013; Brott et al. 2011). As explained by Rímulo et al. (2018, their Sect. 5.2.5 and references therein), Be stars can avert the possible permanent angular-momentum crisis by the viscous decretion of matter and associated angular momentum. Viscosity can enable the formation of a Keplerian disc by redistributing the specific angular momentum of ejected matter to the extent that a $\sim 1 \%$ fraction reaches Keplerian velocities, and the rest falls back to the star (Lee et al. 1991). The variability of the mass content of the disc may provide a means to estimate the amount of angular momentum lost along with the matter (Ghoreyshi et al. 2018; Rímulo et al. 2018).

An obvious alternative mechanism to spin up Be stars is mass transfer in a binary. In fact, in some classical Be stars, hot subluminous companions have been found (Peters et al. 2016; Wang et al. 2018, for other examples see below) so that the high spin rate of the B star may be the result of mass transfer from 
the companion, which initially was the more massive star. The effectiveness of viscous decretion to build Keplerian discs is unaffected by sufficiently distant companion stars. Therefore, viscous decretion is thought to be a universal property of $\mathrm{Be}$ stars, because Be stars with known short orbital periods are very rare (however, it is also possible that the frequency of such systems is reduced if the formation of stable decretion discs is hindered by the companion). For viscous decretion to form Keplerian discs, it must be supplied with matter by a stellar mass-loss process. The ubiquity of nonradial pulsations (NRPs) in Be stars (Rivinius et al. 2013; Baade et al. 2017; Semaan et al. 2018), and the co-phasing of apparent mass-loss events with maxima of the vectorial amplitude sum of multiple pulsation modes (Baade et al. 2018), strongly suggest the need to search for the root of the mass loss in multi-mode NRPs in single, as well as binary, Be stars. Most probably, single- as well binarystar formation channels of Be stars are also realised by nature, either alone or in combination. This paper considers the binary channel only, without implication for the single-star channel.

Since the X-ray luminosity of OB stars is proportional to their bolometric luminosity (Güdel \& Nazé 2009), X-rays caused by shocks in the winds are not an important property of isolated Be stars (Cohen et al. 1997). A possible small X-ray excess in Be stars with regard to normal B stars (Cohen 2000) may be due to additional shocks in the interface region between wind and disc. However, some binary Be stars do reveal themselves through prominent, often strongly modulated, X-ray emission. In the vast majority of these Be X-ray binaries (BeXRBs, Reig 2011), a neutron star accretes matter when it passes through or close to the Be star's circumstellar disc, and part of the gravitational energy released in the accretion process is emitted in the X-ray domain. While the X-ray flux of all BeXRB detected in early surveys is pulsed, systematic searches in nearby galaxies are beginning to identify sources without short periods (Haberl \& Sturm 2016); either they are genuinely aperiodic, or the periods were not found because they are too long to be easily determined.

A second subclass, which accounts for $\sim 1 \%$ of all classical Be stars, is also identified on the basis of X-ray properties (Smith et al. 2016a; Nazé \& Motch 2018). These stars emit unusually hard $(\geq 5-10 \mathrm{keV})$, but only moderately strong $\mathrm{X}$-rays, which are variable on all timescales and distinctly thermal (Nazé \& Motch 2018, see also Sect. 2). While the hardness is not too discrepant from observations of BeXRBs, the X-ray luminosity of accreting BeXRBs is much higher. The prototype of this second subclass is $\gamma$ Cas. Accordingly, the other members are often called $\gamma$ Cas stars. $\gamma$ Cas is also the first Be star that was discovered (Secchi 1866). For this reason, $\gamma$ Cas is considered by some as the prototype of Be stars (for instance the General Catalog of Variable Stars, Samus' et al. 2017, calls all Be stars "GCAS" (or "BE") stars). However, $\gamma$ Cas has a number of observed properties that only few Be stars share (Harmanec 2002) although it is not clear to what extent this is due to the particularly rich database. The most important difference is the mentioned X-ray flux.

Because the X-ray properties of $\gamma$ Cas do not match any conventional category of X-ray sources in early-type stars, Smith and collaborators (see Smith et al. 2016a, for references) have, in a long series of papers, developed the unconventional notion that the X-rays from $\gamma$ Cas result from the interplay between two magnetic fields, one at the stellar surface and the other in the disc. Both are said to be not observationally detectable because of their small spatial scales. Nevertheless, there seems to be the associated hope (Motch et al. 2015) that this model, which in the following will be called the magnetic model for short, may even- tually explain Be stars at large. Because of the small fraction of Be stars with $\gamma$ Cas-like X-ray properties, and the elusiveness of direct observational evidence for the suggested magnetic fields, it is important that no mistake is made with any extrapolating generalisation.

This paper develops a completely different concept to explain $\gamma$ Cas stars that has little implication for the majority of Be stars. It incorporates, without restriction, the above sketch, which is a general picture of classical Be stars, so that $\gamma$ Cas stars are ordinary Be stars with some additional properties. The proposed main difference is the response of the circumstellar Be disc and/or the Be wind to the impact of a fast wind from a heliumstar companion.

For a better understanding, the key properties of the currently most prominent $\gamma$ Cas stars, namely $\gamma$ Cas itself, $\pi$ Aqr, and BZCru, are recalled in Sect. 2. Section 3 describes the magnetic model in more detail as well as the white-dwarf (Tsujimoto et al. 2018) and the magnetic-neutron-star propeller model (Postnov et al. 2017) that were recently proposed as alternatives. Because $\gamma$ Cas and $\pi$ Aqr are binaries, the role of binarity in $\mathrm{Be}$ stars is reviewed in the context of extent observations (Sect. 4) and evolutionary models (Sect. 5). Conclusions are given in Sect. 6.

\section{Observed properties of $\gamma$ Cas stars}

\subsection{Overview}

At this moment, a Be star is admitted to the $\gamma$ Cas family on the basis of its X-ray flux, the latter should be hard $(L(2-10 \mathrm{keV}) / L(0.5-2 \mathrm{keV})>1.6)$, moderately strong $\left(\log \left(L_{\mathrm{X}} /\right.\right.$ $\left.L_{\text {bol }}\right) \sim-6$ ), and thermal (Nazé \& Motch 2018). These selection criteria have mostly identified stars in the narrow spectral-type range of B0.5-B1.5 (with luminosity classes V-III), although some exceptions are beginning to be reported (Nazé \& Motch 2018). The $0.1-10 \mathrm{keV} \mathrm{X}$-ray luminosity is intermediate between noninteracting Be stars and BeXRBs. Table 1 reproduces the main properties of the $\sim 15$ currently known $\gamma$ Cas stars as compiled by Nazé \& Motch (2018). A very useful account of the X-ray properties of $\gamma$ Cas stars and possibly related objects is available from Tsujimoto et al. (2018).

The individual characteristics of the three best-observed representatives are outlined in the following subsections.

\section{2. $\gamma$ Cas}

After the first detection of X-rays from $\gamma$ Cas (Jernigan 1976), there was not much of an alternative to a classification as a BeXRB. However, the lack of pulsing (Parmar et al. 1993) and regularly repeating X-ray outbursts when a putative compact companion would, in its (eccentric) orbit, accrete matter from the Be disc (Okazaki \& Negueruela 2001), casts doubts on the origin of the X-rays, and the nature of $\gamma \mathrm{Cas}$ $(=$ HR $264=$ HD $5394=$ HIP 4427) has been controversial ever since.

$\gamma$ Cas was also one of the first Be stars in which discrete absorption components (DACs) of UV resonance lines were discovered (Henrichs et al. 1983). DACs are nearly universal in luminous OB stars (Howarth \& Prinja 1989), and usually attributed to corotating interaction regions in the wind that originate from the high intrinsic instability of the wind, perhaps triggered by photospheric inhomogeneities (Cranmer \& Owocki 1996). The azimuthal propagation of the interaction regions may lead to a modulation of X-ray flux resulting from shocks in the 
N. Langer et al.: $\gamma$ Cas stars: Normal Be stars with discs impacted by the wind of a helium-star companion?

Table 1. Key observational data for known $\gamma$ Cas stars taken from Nazé \& Motch (2018, their Table 5).

\begin{tabular}{|c|c|c|c|c|c|c|c|c|}
\hline No. & Name & Spectral type & $\log \left(L_{\mathrm{X}} / L_{\mathrm{bol}}\right)$ & $\begin{array}{c}L_{\mathrm{X}} \\
10^{31} \mathrm{erg} \mathrm{s}^{-1}\end{array}$ & $\begin{array}{c}L_{\mathrm{X}, \mathrm{hard}} \\
10^{31} \mathrm{erg} \mathrm{s}^{-1}\end{array}$ & Hardness ratio & $\begin{array}{c}\mathrm{kT} \\
\mathrm{keV}\end{array}$ & $\begin{array}{c}v \sin i \\
\mathrm{~km} \mathrm{~s}^{-1}\end{array}$ \\
\hline 1 & $\gamma \mathrm{Cas}$ & BoIV-Vpe & -5.39 & 85.0 & 65.1 & 3.25 & $14-25$ & 295 \\
\hline 3 & V782 Cas & B2.5III:[n]e+ & -5.25 & 30.3 & 29.9 & 63.1 & 7 & \\
\hline 17 & PZ Gem(high) & O9pe & -6.14 & 9.66 & 7.87 & 4.32 & 16 & 265 \\
\hline 26 & HD90563 & $\mathrm{B} 2 \mathrm{Ve}$ & -5.85 & 32.0 & & & & \\
\hline 34 & BZ Cru & B0.5IVpe & -5.69 & 27.6 & 20.3 & 2.81 & 13 & 338 \\
\hline 37 & HD119682 & Bove & -5.63 & 66.7 & 47.9 & 2.55 & $8-17$ & 200 \\
\hline 39 & V767 Cen & $\mathrm{B} 2 \mathrm{Ve}$ & -5.37 & 26.2 & 17.4 & 1.97 & 6 & 100 \\
\hline 40 & CQ Cir & $\mathrm{B} 1 \mathrm{Ve}$ & -4.30 & 175 & 147 & 5.26 & 9 & 335 \\
\hline 47 & V759 Ara & B2Vne & -5.29 & 41.9 & 31.9 & 3.21 & 10 & 277 \\
\hline 51 & V3892 Sgr & $\mathrm{Oe}$ & -5.78 & 30.8 & 21.2 & 2.24 & $7-14$ & 260 \\
\hline 53 & V771 Sgr & B3/5ne & -4.64 & 24.5 & 21.1 & 6.29 & 8 & \\
\hline 54 & HD316568 & B2IVpe & -6.26 & 4.04 & 2.43 & 1.60 & $4-6$ & \\
\hline 75 & V2156Cyg & B1.5nnpe & -5.30 & 7.53 & 6.51 & 6.34 & 3 & \\
\hline 79 & $\pi \mathrm{Aqr}$ & $\mathrm{B} 1 \mathrm{Ve}$ & -5.59 & 7.44 & 5.80 & 3.56 & 12 & 243 \\
\hline 83 & V810 Cas & B1npe & -5.14 & 48.4 & 41.1 & 5.58 & 64 & 422 \\
\hline
\end{tabular}

Notes. Soft and hard X-ray fluxes refer to the $0.5-2.0 \mathrm{keV}$ and $2.0-10.0 \mathrm{keV}$ intervals, respectively.

wind (Oskinova et al. 2001). Because of their ubiquity in luminous stars with radiatively driven winds, the DACs in $\gamma$ Cas do not reveal anything specific about the properties of this star, except that its mass-loss process and wind are perfectly normal for an early-type Be star.

Four periods have been reported for $\gamma$ Cas and used in various attempts to identify the nature of this star's X-ray activity. The orbital period of $\sim 203.5 \mathrm{~d}$ was first identified by Harmanec et al. (2000). Later refinements revised the eccentricity to $\sim 0$ and are based mainly on radial-velocity measurements of the flanks of the $\mathrm{H} \alpha$ emission-line profiles (Miroshnichenko et al. 2002; Nemravová et al. 2012). Although major long-term corrections are required, and the radial velocity of the disc is not the same as that of either of the two stars, neither the value of the period nor its nature are disputed. The orbital period was also found in the temporarily flat top of the $\mathrm{H} \alpha$ emission-line profile (Borre et al., in prep.), which is probably orbitally modulated by the interaction of the companion with the (spiral) disc structure (cf. Panoglou et al. 2018). Harmanec et al. (2000) propose a likely mass range of the primary ${ }^{1}$ between 13 and $18 M_{\odot}$.

The nature of the companion is not well-constrained. The mass is about one solar unit, and Nemravová et al. (2012) suggested that it might be a helium star. Miroshnichenko et al. (2002) find inhomogeneities in the disc and consider one possible explanation to be that $\mathrm{H} \alpha$-emitting material is associated with the secondary. In search for a spectral signature of the secondary, Wang et al. (2017) cross correlated the UV spectrum with model sdO spectra. However, this effort failed, because the very hot primary dominated the cross-correlation function, which, moreover, is very broad due to the rapid rotation of the B-star primary. Probably because of the unfavourable magnitude difference at the wavelengths used, long-baseline $\mathrm{H} \alpha$ (Tycner et al. 2006) and $K$-band (Gies et al. 2007) interferometry have not detected the companion either. However, the circumstellar disc was resolved, and the derived inclination angles of $55^{\circ}$ and $51^{\circ}$, respectively, are in very good agreement.

For 15 years, a $1.216 \mathrm{~d}$ period was seen in single-site groundbased photometry (Henry \& Smith 2012), but eventually dropped below the detection threshold of very few mmag. Both the fre-

1 With primary, we designate the brighter of the two stars in a binary. quency and the decay in amplitude of this second periodic variability were also found in SMEI space photometry (Borre et al., in prep.). Later space photometry with BRITE-Constellation confirmed the absence of the $1.216 \mathrm{~d}$ period at the $2-3 \mathrm{mmag}$ level (Baade et al. 2017; Borre et al., in prep.). Instead, BRITE detected a very nearly, but probably not perfectly, three times shorter third period at $0.403 \mathrm{~d}$ (frequency: $2.48 \mathrm{c} / \mathrm{d}$ ) with a peak-to-peak amplitude slowly varying between $\sim 2$ and $\sim 9$ mmag (Baade et al. 2017; Borre et al., in prep.). A fourth frequency was identified at $1.25 \mathrm{c} / \mathrm{d}$ in the SMEI observations (Borre et al., in prep.). An attempt was made to use Doppler shifts of the $2.5 \mathrm{c} / \mathrm{d}$ frequency to locate the site of the variability in the system. However, the time baseline of the BRITE data was too short, and the systematic noise of the SMEI observations was too large (Borre et al., in prep.).

The long-term constancy of the three short periods implies either rotation or pulsation as their origin. Rotationally-induced variability with period $P$ would require some physical property to vary along the star's circumference with an azimuthal scale of $\left(P / P_{\text {rot }}\right) \times 2 \pi$. For instance, temperature, abundances, or magnetic structures. There is no such report for $\gamma$ Cas (apart from the optical broad-band flux). Radial pulsations are not known in Be stars, but both short periods are well within the range of NRPs found in other Be stars (Rivinius et al. 2016; Baade et al. 2017; Semaan et al. 2018). Since the 1.216 d variability faded while the $0.403 \mathrm{~d}$ variability rose, it is plausible to believe that both are of the same nature, which can, then, only be NRPs. In fact, space photometry (Rivinius et al. 2016; Baade et al. 2017; Semaan et al. 2018) has detected multiple low-order NRP modes in many Be stars over the full range of B-type stars.

Ongoing and forthcoming large-scale photometric surveys from space will show how typical (multi-mode) NRP is for $\mathrm{Be}$ stars. If the pulsation properties of $\mathrm{Be}$ stars are different from those of Bn stars (very rapidly rotating B-type stars identified through their equator-on orientation, but not known to have exhibited emission lines, i.e. not possessing a circumstellar disc), this would be a strong indicator that NRPs are a defining property of Be stars, probably through their involvement in mass-loss events feeding the disc.

Additional periods may be hidden in complex spectroscopic line-profile variability. In agreement with quite similar observations in other early-type stars, Yang et al. (1988) and 
Horaguchi et al. (1994) attributed such variability in optical absorption lines also of $\gamma$ Cas to high-order NRP. Intermediateto higher-order NRP modes were also deduced from long series of spectra of other Be stars (e.g., Reid et al. 1993; Kambe et al. 1997), including $\pi$ Aqr (Peters \& Gies 2005). Smith et al. (2016a) rejected the NRP hypothesis for $\gamma \mathrm{Cas}$, because they found the variations of UV lines to be erratic, and each migrating subfeature in the line profiles to maintain its identity for no more than a very small number of hours.

However, the $30 \mathrm{~h}$, meaning, only about one rotational period, of HST spectroscopy considered by Smith et al. (2016a) are without doubt insufficient for the proper tracking of features with similar but different propagation rates, and for the determination of their periods. Accordingly, the suggestion by Smith et al. (1998) that the subfeatures are only rotationally advected is lacking a solid observational foundation. By contrast, Walker et al. (2005) observed $\zeta$ Oph (O9.5 Ve) for 24 days with the MOST space photometer and during 17 of these 24 days with three spectrographs at different geographical longitudes. They detected at least a dozen photometric, and eight spectroscopic periods. Six periods were in common with both datasets and interpreted as intermediate-order NRPs. An obvious rotation period was not identified, and the multi-periodicity of the migrating subfeatures rules out the rotational hypothesis for them.

In addition to the three genuine periods in $\gamma$ Cas, there are also cyclic optical broad-band flux variations on seasonally changing timescales around $70 \mathrm{~d}$, with a total range of 50-91 d. The peakto-peak amplitude of $\sim 0.02 \mathrm{mag}$ is not too far from the sensitivity threshold to so slow variations of single-site groundbased photometry. Robinson et al. (2002) combined the earlier cycles into a single sinusoid with adaptive period and compared this variablestretch pseudo-sine curve of optical light to the X-ray flux. They derived a correlation in the variability of the two domains using only two photometric seasons and just six epochs of X-ray data. Since the pseudo-sine curve interpolates the light curve, the effective comparison is between seasonally fragmentary optical-flux and very patchy X-ray observations. There is no assurance that such a data treatment can lead to a stress-resistant conclusion.

From just one day of simultaneous X-ray and UV observations, Smith and collaborators (for references see Smith et al. 2016a) inferred correlations between X-ray flux on one side, and UV flux, UV spectral lines, etc. on the other. However, it is not clear that coincidences of two features each in two short datasets can carry high weight in an object that in all observed wavelength regions is variable on all timescales. More significant is the correlation over 15 years between X-ray and optical flux reported by Motch et al. (2015), although it is not clear which effect the choice of the time windows has. From their comparison, these authors conclude that the X-rays lag the optical flux by no more than a month. Because the radial drift velocity of matter in $\mathrm{Be}$ discs is only of the order of a few $\mathrm{km} \mathrm{s}^{-1}$ (Rivinius et al. 1999), the time delay of X-ray emission due to accretion by a companion at an au-scale distance would be much longer. By contrast, a lag of only a month is more plausible if it takes a month for the disc to build up and the interaction between the two postulated magnetic fields to commence.

However, in the cross-correlation function, there is a broad and not well separated peak near three years. In view of this network of claimed correlations, it is surprising that the purported rotation period has not been seen modulating any observable (other than the optical flux).

Lopes de Oliveira et al. (2010) emphasised the need for multi-component fits of the continuum X-ray flux distribution. From high-spectral-resolution XMM-Newton observations with a complex emission-line spectrum, they derived opticallythin thermal emissions at four discrete temperatures, namely $12-14 \mathrm{keV}$, perhaps at $2.4 \mathrm{keV}$, and with confidence at 0.6 and $0.11 \mathrm{keV}$. From observations between 0.6 and $100 \mathrm{keV}$, Shrader et al. (2015) firmly rule out any power-law component and thereby confirm the thermal nature of the X-ray flux. Smith et al. (2012a) report that after an apparent mass-loss event (ejection of matter into the disc), an absorbing layer developed temporarily, indicating the presence of additional matter along the line of sight. Temperature contrasts are also evidenced by spectral lines (Lopes de Oliveira et al. 2010; Smith et al. 2012a).

Using independent observations, Tsujimoto et al. (2018) basically agree with the stated decomposition of the X-ray continuum. They also confirm that changes in the hardness ratio are only weakly coupled to flux variations, which mainly occur in the hottest plasma above $4 \mathrm{keV}$, while the softer X-rays are more stable and are most of the time only negligibly absorbed. A new finding though are dips in softness, especially of the ratio [0.5-2 keV]/[4-9 keV], which last a few ks. Because these dips are unrelated to flux increases in the hard band, Tsujimoto et al. (2018) conclude that these fadings are caused by absorption in temporarily intervening matter. This is consistent with the similar picture derived by Smith et al. (2012a) from X-ray observations during an outburst of the $\mathrm{B}$ star. Adopting the outburst interpretation, it seems plausible that the X-ray-emitting volume was (partly) located behind the ejecta. The implied proximity to the B star would argue, as may be deduced from the time delays between optical and X-ray fluxes, against the X-rays forming near a companion star at an au-scale distance.

In the latest of his papers on $\gamma$ Cas, Smith (2019) discusses various observations once again, offering basically the same interpretations. It seems useful to point out that all the old observations were obtained with instruments not employing solid-state detectors. In those detectors, photons do not merely excite electrons (internal photoelectric effect), but lead to the physical emission of electrons (external photoelectric effect), which are subsequently amplified and measured. As a result, measurements can in some cases deviate more from unbiased photon statistics than is typical of solid-state detectors. Moreover, physically-emitted electrons are more susceptible to subtle external perturbations.

\section{3. $\pi$ Aqr}

On the basis of its X-ray properties, Nazé et al. (2017) recently classified $\pi$ Aqr $\quad(=$ HR $8539=$ HD $212571=$ HIP 110672) as another $\gamma$ Cas star. The similarity concerns not only the X-ray flux and hardness, but also the variability. During the $50 \mathrm{ks}$ observations with XMM-Newton, several brightenings with a base width of 1-2 ks occurred with pronounced peaks, reaching roughly thrice the previous or subsequent level. As in $\gamma$ Cas, no BeXRB-like outbursts have been observed.

After $\gamma$ Cas itself and BZCru (Sect. 2.4), $\pi$ Aqr became the third $\gamma$ Cas star in the Bright Star Catalog (and is moreover equatorial), so that for $\pi$ Aqr, a good record of its general properties and variability in other wavelength regions is also available. Wisniewski et al. (2010) documented the long-term stability of the disc orientation in space by spectropolarimetry. The decreasing $\mathrm{H} \alpha$ emission strength traced the dissipation of the disc over nearly a decade. Variations in $\mathrm{H} \alpha$ equivalent width and continuum polarisation also caught a number of outbursts (Wisniewski et al. 2010) which are quite typical, especially of early-type Be stars (Labadie-Bartz et al. 2018; Bernhard et al. 2018). The occurrence of DACs in UV wind lines (Smith 2006) is also common among Be stars (Grady et al. 1989). 
Bjorkman et al. (2002) found that $\pi$ Aqr is an $84.1 \mathrm{~d}$ binary. The mass ratio is about $6: 1$, which should be more favourable for the detection of the companion than the $\sim 15: 1$ ratio in $\gamma$ Cas. Depending on the inclination angle, the mass of the secondary may be between 2.2 and $4.5 M_{\odot}$. The orbital motion of the secondary was derived from a "travelling emission component", which the authors attributed to a gaseous envelope surrounding the secondary. From $\mathrm{H} \alpha$ profiles covering $~ 40$ orbits, Zharikov et al. (2013) extracted the same period for the violetto-red ratio $V / R$ of the two emission peaks. Accordingly, the disc structure is phase-locked to the position of the companion. The power spectrum plotted by Zharikov et al. (2013) does not include the first harmonic. If this omission is justified, it would mean that any two-armed spiral structure (Panoglou et al. 2018) is not axisymmetric, perhaps because one arm strongly dominates (or the two arms are not $180^{\circ}$ apart in disc azimuth). In fact, the study identifies an extended region of enhanced $\mathrm{H} \alpha$ line emission between the two stars. The strength of this emission follows the long-term variability of the overall emission strength. As for $\gamma$ Cas, the cross-correlation technique of Wang et al. (2017) did not detect the companion to this hot and broad-lined star.

Nazé et al. (2017) put forward the argument that the secondary in the $\pi$ Aqr system is not a compact object itself, and that no such third body is likely to be in a closer orbit than the secondary. Therefore, they conclude that the X-ray properties of $\pi$ Aqr and, by implication, $\gamma$ Cas stars in general are not caused by a compact companion. However, if the interaction of the companion with the disc leads to additional $\mathrm{H} \alpha$ emission (see also Bjorkman et al. 2002), more power seems required than is available from an intermediate-mass main-sequence star.

As in $\gamma$ Cas and several other Be stars, NRPs of intermediate degree $(m=5)$ have been deduced from the photospheric lineprofile variability of $\pi$ Aqr (Peters \& Gies 2005). Rivinius et al. (2003) observed low-order line-profile variability not matching the quadrupole NRP patterns typically seen in Be stars.

\subsection{BZCru}

The X-ray similarity to $\gamma \mathrm{Cas}$ of BZCru $(=\mathrm{HR} 4830=$ HD 110432 = HIP 62027) was established by Smith et al. (2012b). In six visits by the Rossi X-ray Timing Explorer (RXTE), each collecting 8-9h of observations with the Proportional Counter Array (Jahoda et al. 1996), well over 1000 flares were seen by the authors. With $5 \mathrm{~s}$ binning, they could be as short 2.5 bins, and, in some cases, lasted more than a minute, with an average rate of about one flare in twenty $5 \mathrm{~s}$ bins, which is not far from the confusion limit. As in $\gamma$ Cas, most of the time, the hardness ratio did not change during the flaring. On two occasions, the X-ray emission subsided for a few hours. The six datasets span only 155 days, yet, the authors derived a "period" of 226 days. Tsujimoto et al. (2018) also applied their models to BZCru. As in the case of $\gamma$ Cas, they achieved satisfactory fits of the X-ray flux distribution, but could not distinguish between a nonmagnetic and a magnetic white dwarf.

As most other Be and supergiant OB stars with stellar winds, BZCru exhibits variable DACs (Smith et al. 2012b). The same authors also found intermittent migrating subfeatures in stellar line profiles, which, in other OB and Be stars, were attributed to nonradial pulsation, but did not report periods. They speculated about "magnetically confined clouds" but admitted that this is "not proven". As mentioned above (Sect. 2.2), such speculations were disproven in the case of $\zeta$ Oph (O9.5 Ve, Walker et al. 2005).

Smith \& Balona (2006) also noted that if BZ Cru is a member of NGC 4609, it would be a blue straggler. From a ded- icated interferometric search, Stee et al. (2013) only derived upper detection limits for a companion star. The disc had a strongly asymmetric structure the nature of which could not be firmly established. Wang et al. (2018) did not detect the signature of an $\mathrm{sdO}$ companion in International Ultraviolet Explorer (IUE) UV spectra.

\subsection{Synopsis}

Any attempt to extract commonalities from a sample of just three, albeit well-studied, representatives must appear presumptuous. However, relying on Nazé \& Motch (2018) for the X-ray properties of $\gamma$ Cas stars, the following working description is perhaps broadly agreeable: (1) Typical spectral subtypes fall into the range B0.5-B1.5. (2) The X-ray flux is hard $(L(2-10 \mathrm{keV}) / L(0.5-2 \mathrm{keV})>1.6)$, moderately strong $\left(\log \left(L_{\mathrm{X}} / L_{\mathrm{bol}}\right) \sim-5.5\right)$, and thermal. (3) The X-ray flux is variable on timescales from seconds to years. (4) Variations in the $\mathrm{X}$-ray hardness ratio are small and mainly down to the hard component. (5) There are occasional reductions in the soft X-ray flux, consistent with intervening absorbers. (6) At least on long timescales, X-ray and optical flux variations track each other. (7) There is a lower-mass and optically-faint companion (may not be the case for BZCru). (8) The companion interacts with the Be disc. (The not finally explained strong asymmetry of the disc of BZ Cru may be caused by a not otherwise detected companion). (9) Intervening absorbers ejected by the B star may localise the $\mathrm{X}$-ray-forming region near the $\mathrm{B}$ star, not around the companion.

\section{Current models for $\gamma$ Cas stars}

\subsection{The magnetic model}

In addition to most of the observed properties listed in Sect. 2.5, the magnetic model rests on the assumptions that (i) the $1.216 \mathrm{~d}$ period of $\gamma \mathrm{Cas}$ is the rotation period of the $\mathrm{B}$ star, (ii) there is a correlation without major relative shift in time between $\mathrm{X}$-ray and optical flux, (iii) there is a correlation, without offsets in time, between X-ray flux and spectral UV features, (iv) the migrating subfeatures in spectroscopic line profiles are not caused by nonradial pulsation, and (v) companion stars are irrelevant for the understanding of $\gamma$ Cas stars. As seen in Sect. 2, all of these assumptions meet with various degrees of doubt and cannot be proved or disproved using currently available observations of $\gamma$ Cas stars.

The magnetic model casts these assumptions into the notion of magnetic fields as the common umbrella. Two kinds of magnetic field are envisioned. One resides in the star and is said to arise from sub-surface convection zones (Cantiello \& Braithwaite 2011). The other one is considered to result from the amplification by magneto-rotational instability (MRI; e.g. Sano et al. 2000) of seed fields in the disc. Circumstellar and stellar magnetic field lines are assumed to temporarily connect via fingers extending from the disc towards the star. Because of the different rotation rates of star and disc, the field lines are thought to be stretched, eventually disrupted, and, finally, reconnected. The snapping back of the field lines is suggested to accelerate charged particles to high energies dissipated as X-rays when they hit the star. Disc instabilities and mass injections from the star are seen as the origin of the assumed correlation, without much delay, between optical and X-ray flux on the variable $70 \mathrm{~d}$ timescale. Migrating subfeatures in absorption lines are attributed to superphotospheric cloudlets forced into corotation by the putative magnetic field (Smith et al. 1998). As discussed in Sect. 2.2, the empirical basis for this latter belief is deficient. 
According to Smith et al. (2017), neither the stellar nor the disc magnetic field postulated by them is directly observable, because their structures are thought to be too tangled and smallscale. Therefore, the magnetic model is not, a priori, in direct conflict with a survey of $85 \mathrm{Be}$ stars (incl. $\gamma$ Cas, $\pi$ Aqr, and BZ Cru Wade et al. 2016; Neiner et al., in prep.) that did not find one star with a large-scale magnetic field, whereas for nonBe early-type stars in the same survey, the typical fraction of magnetic stars is about 5-10\% (Wade et al. 2016). (A possible explanation of this negative result is that a magnetic field would destroy a Keplerian disc (ud-Doula et al. 2018) so that magnetic Be stars cannot exist. By contrast, rapid rotation and a magnetic field are not strongly mutually exclusive for B0.5 to B1.5 stars near the zero age main sequence). In spite of the lack of detected large-scale magnetic fields in Be stars, the stellar magnetic field in $\gamma$ Cas stars is also believed to be responsible for the claimed rotational modulation with an extremely constant period of the optical broad-band flux (Smith et al. 2016b), which requires a large-scale structure that does not migrate in the corotating frame. The magnetic model does not address this obvious tension other than by hypothesising that the non-detection of a magnetic field is related to the disappearance of the photometric $1.215 \mathrm{~d}$ variability, and a result of the decay of the stellar magnetic field (Smith 2019). If so, $\gamma$ Cas would, during the presence of the $1.215 \mathrm{~d}$ period, have possessed a large-scale magnetic structure not seen in any other Be star.

The two magnetic constructs are imported from other contexts. Convective sub-surface dynamos might produce variable inhomogeneous surface brightness distributions that otherwise, in purely radiative atmospheres, lack a simple explanation. MRI is broadly invoked to produce the level of viscosity needed to bring the timescales of accretion processes into agreement with observational constraints (Martin et al. 2019). (It is useful to note that the viscous-decretion-disc model for Be stars [cf. Sect. 1] also merely assumes viscosity but does not explain it). However, to date, neither magnetic-field-producing mechanism seems to have found direct observational confirmation, even in the domains they were designed for. This motivates searches for alternate explanations of the $\gamma$ Cas stars.

The magnetic model was conceived before the (optically) faint and low-mass companion star of $\gamma$ Cas was discovered. The model has evolved over the years. However, also in its current version, it does not foresee any role for the companion to contribute to the observed phenomena. This attitude is seemingly reinforced by the fact that BeXRBs, where compact companions are the main X-ray actors, and $\gamma$ Cas stars are clearly distinguished populations. If faint low-mass companions in low-eccentricity orbits are characteristic of $\gamma$ Cas stars, a large difference in the X-ray properties of BeXRBs and $\gamma$ Cas stars may be expected, because in near-circular orbits, the Be disc is strongly truncated. As a result, the disc remains well within the B star's Roche lobe so that major X-ray outbursts with the orbital period are unlikely (Okazaki \& Negueruela 2001). Therefore, accreting binary models of $\gamma$ Cas stars need to be powered by the B star's mass loss.

\subsection{Accretion onto a white dwarf}

One way of avoiding the overproduction in $\gamma$ Cas stars of X-rays at the level of BeXRBs is to assume a white dwarf (WD) as the accreting body, because it has a shallower gravitational potential than that of a neutron star or a black hole. This was first proposed by Haberl (1995). In fact, accreting white dwarfs in novae and symbiotic stars are X-ray sources of roughly comparable properties. Contrary to the purely parametric formalisms of most ear- lier studies, Tsujimoto et al. (2018) employed models specifically designed for white dwarfs accreting matter from a cool companion, such as in novae or symbiotic stars, and included reflection by the white dwarf of X-rays as well as absorption. They achieved reasonable fits of the X-ray flux distributions of both $\gamma$ Cas and BZ Cru. However, the models could not conclusively discriminate between magnetic (as in polars or intermediate polars) and nonmagnetic (as in [dwarf] novae) WD companions.

Hamaguchi et al. (2016) offered the interesting idea that the cooler X-ray-emitting plasma "probably originates from the Be primary stellar wind, while the hot component may originate from the head-on collision of either the Be or WD wind with the Be disc". In a different context, it has, in fact, been shown that Be discs are probably subject to ablation by the B star's radiation (Kee et al. 2018, and references therein). However, if an interaction between the wind from a Be star with the disc were at the origin of the hard X-rays from $\gamma$ Cas, more than just $\sim 1 \%$ of the Be stars would be $\gamma$ Cas stars. A variant of the suggestion of a collision with a wind from a companion is developed in Sect. 5.

The mass estimate for the companion to $\gamma$ Cas of one solar mass is at the high end of WDs. However, if the range of 2.2-4.5 $M_{\odot}$ for the secondary star in $\pi$ Aqr (Bjorkman et al. 2002) is correct, the WD model would not be applicable. Depending on how much mass is transferred back to a WD companion during the later evolution of the B-type primary, and when this happens, such systems might even be progenitors of a thermonuclear Type Ia supernova explosion of the WD and a core-collapse Type II supernova of the B star.

\subsection{The propeller model}

Recently, Postnov et al. (2017) advanced the so-called propeller model, which employs a neutron star, but reduces the X-ray flux from direct accretion, like in BeXRBs, by letting the magnetic field and rapid rotation of the neutron star suitably moderate the accretion rate. Moreover, because the X-ray emission is from a hot halo, it is not rotationally pulsed (as observed). This construct would seem to eliminate the discrepancy in the X-ray domain between $\gamma$ Cas stars and BeXRBs. However, Smith et al. (2017) have nevertheless vehemently rejected the propeller model. In particular, they argue that the X-rays form close to the B star, not near the companion at au-scale distances, because of intermittent X-ray attenuations by cold plasma, ejected by the B star, between the X-ray-emitting region and the observer. Furthermore, they insist that the density of the X-ray emitting plasma is of order $10^{15} \mathrm{~cm}^{-3}$, meaning, at a photospheric level, while the propeller model yields values near the inner radius of the magnetosphere that are 1-2 orders of magnitude lower.

In addition, the assumption of neutron-star companions to $\gamma$ Cas and $\pi$ Aqr is not straightforward. Because BeXRBs can exist for a few $10^{6}$ years after the supernova explosion that formed the neutron star, whereas the remnant nebulae merge with the interstellar medium within a few $10^{3}$ years, the absence of such nebulae around these stars is not an obstacle to the neutron-star hypothesis. Better indicators are, however, their orbital eccentricity and the space velocity, both of which may be significantly modified by a supernova explosion. This is briefly discussed in the following two subsections.

\subsubsection{Impact of a supernova explosion on orbital eccentricity}

If a star exploding in a binary experiences a significant kick, the eccentricity of the orbit grows, and the plane of the orbit may get tilted with respect to the equatorial plane of the previous 


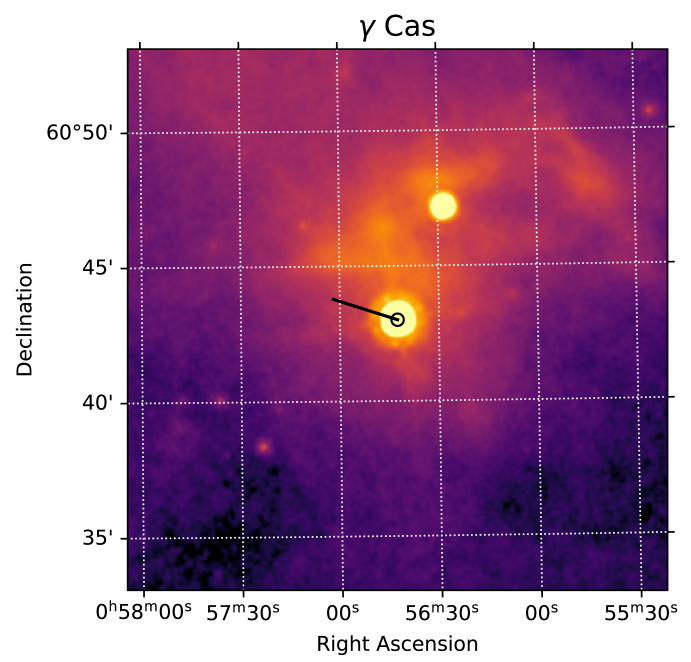

Fig. 1. WISE $24 \mu \mathrm{m}$ image of $\gamma$ Cas. The black line at the centre illustrates the 10000-year proper motion (corrected for Galactic rotation) as measured by HIPPARCOS ( $\gamma$ Cas is too bright for Gaia DR2).

mass gainer, which, in the case of Be stars, is also the plane of the disc. The details depend very much on the direction of the kick (Renzo et al. 2019). These expectations find their confirmation in many observed BeXRBs (Reig 2011). They do not appear to be satisfied in $\gamma$ Cas (Gies et al. 2007) and $\pi$ Aqr (Bjorkman et al. 2002; Zharikov et al. 2013) the orbits of which seem nearly circular. Postnov et al. (2017) invoke an electroncapture supernova explosion for the progenitor of the assumed neutron star. Such explosions are thought to impart a low kick on the remnant. However, about $10 \%$ of the rest mass of the exploding star is lost as neutrinos. Even if this mass loss is symmetric with regard to the centre of gravity of the exploding star, it is asymmetric at the binary's centre of gravity, and so imposes some orbital eccentricity on binaries that remain bound.

\subsubsection{Impact of a supernova explosion on space velocity}

If a supernova explosion increases the velocity of a binary relative to the ambient interstellar medium (ISM), a bow shock may develop when a stellar wind impacts the ISM. A prototypical case is the O9.5 Ve runaway star $\zeta$ Oph (del Valle \& Romero 2012, and references therein). However, as Renzo et al. (2019) explain, most surviving systems are not expected to be accelerated by more than $\sim 30 \mathrm{~km} \mathrm{~s}^{-1}$.

Bodensteiner et al. (2018) inspected and classified WISE (Wright et al. 2010) $24 \mu \mathrm{m}$ images of all OBA stars in the Bright Star Catalog (Hoffleit \& Jaschek 1991), including $\gamma$ Cas and $\pi$ Aqr. WISE images of the regions around $\gamma$ Cas and $\pi$ Aqr are reproduced in Figs. 1 and 2. The superimposed proper-motion vectors illustrate the classifications by Bodensteiner et al. (2018) for $\gamma$ Cas and $\pi$ Aqr, respectively. A bow shock can be seen to be associated with $\pi$ Aqr (see also Mayer et al. 2016). However, the apex of the nebula is not aligned with the proper-motion vector. Accordingly, the relative velocity of $\pi$ Aqr and the ambient interstellar medium is not dominated by the stellar motion. $\gamma$ Cas is also surrounded by a nebula, which may be related to the star. But the morphological evidence is weak so that the entry in Bodensteiner et al. (2018) is "not classified". Nevertheless, the peculiar space velocities are close to $\left(\pi\right.$ Aqr: $\left.21 \mathrm{~km} \mathrm{~s}^{-1}\right)$ or even well within ( $\gamma$ Cas: $38 \mathrm{~km} \mathrm{~s}^{-1}$, Bodensteiner et al. 2018) the domain of single run-away stars (Renzo et al. 2019).

The environments of the other $\gamma$ Cas stars in Table 1 were also inspected in the WISE $24 \mu \mathrm{m}$ atlas. However, no convincing

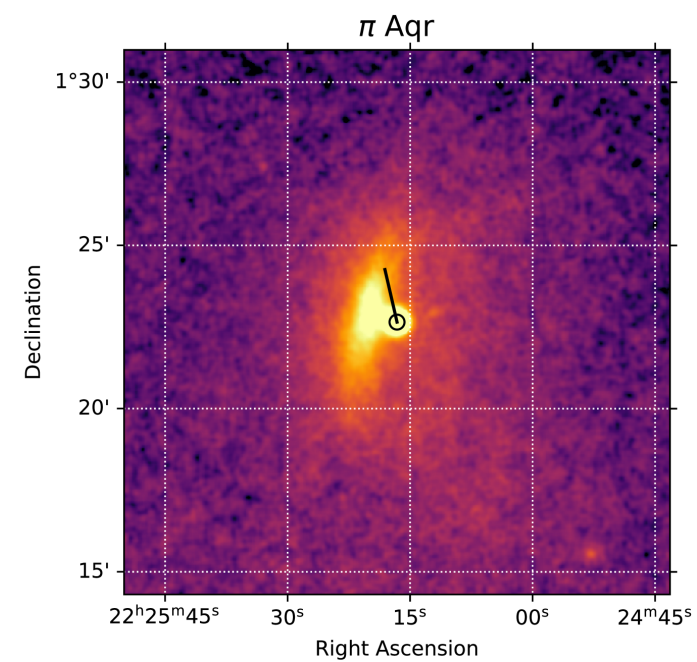

Fig. 2. Same as Fig. 1 except for $\pi$ Aqr and a combination of the HIPPARCOS and Gaia proper-motion measurements.

association of any of these stars with a nebula was found. In most cases, the most likely explanation is the much larger distance implied by the much lower optical brightness. The field around BZ Cru has a very patchy background, with no structure centred on the star standing out.

In summary, there is only mild dynamic or kinematic support of the neutron-star hypothesis for the companions to $\gamma \mathrm{Cas}$ and $\pi$ Aqr. This makes it useful to study, in more depth, the role of binarity at large in the genesis of Be stars from an observational (Sect. 4) as well theoretical (Sect. 5) perspective.

\section{Observations of binary Be stars}

It is not known whether all $\gamma$ Cas stars are binaries. In view of the strongly rotationally-broadened spectral lines of Be stars and the large mass and (optical) luminosity difference between early-type B stars and highly evolved companion stars, attempts to prove definitively that a given Be star does not have such a companion appear illusionary. More quantitative statistical constraints, especially for less evolved systems, may result from possible discoveries of eclipsing systems by large-scale photometric monitoring surveys such as OGLE (Soszynski et al. 2005) or TESS (Ricker et al. 2016). In any event, the assumption of a binary nature of $\gamma$ Cas stars is not currently in obvious conflict with the available observational evidence.

Early suggestions for a possible binary origin of Be stars were made by Kř́ž \& Harmanec (1975) and Pols et al. (1991), triggering various observational searches. The former work assumed that the Be discs are accretion discs. However, owing to the lack of accreting classical Be stars, the observational support is, at best, weak. Since a decretion disc can only be observed after the mass transfer, Be stars formed by mass transfer are expected to have stripped companions that cannot fill their Roche lobes, or their compact remnants.

Sometimes, it is even asked whether all Be stars have highlyevolved companions (e.g. Wang et al. 2017), making their Be-typical rapid rotation the result of mass transfer from their progenitors (cf. Introduction). Certainly, the scarcity of Be stars with main-sequence companions shows that, if a Be star is double, its companion is, very probably, highly evolved. From a very elaborate study based on the comparison of kinematic data from Gaia for a large sample of Be stars and detailed modelling, Boubert \& Evans (2018) infer that the $13.1 \%$ fraction of 
runaway stars found by them is probably sufficient to conclude that all Be stars are post-mass-transfer binaries. However, the apparent preference for lower-mass and highly evolved companions may bias the result if Be stars with relatively close and/or more massive companions cannot maintain a major stable disc.

\subsection{Highly evolved companions}

In analyses of observations of individual Be stars, neutron-stars, WDs, and sdO companions have, up to now, been considered almost exclusively. The results are briefly summarised in the next three subsections. This overview may soon require completion for helium stars (see Sect. 5).

\subsubsection{White dwarfs}

The first to propose that the remains of the mass donors in $\mathrm{Be}$ star-forming binaries are WDs were Waters et al. (1989) and Pols et al. (1991). Theoretical estimates of the fraction of Be stars with a WD companion reach at least 70\% (Raguzova 2001). Several surveys have been conducted, but no positive detection was made (Meurs et al. 1992), with some authors considering $\gamma$ Cas as the best candidate. Perhaps a formal non-Be star, namely Regulus, currently comes closest to such systems, considering the late spectral subtype (B7V) and the intermittency of Be phases especially among late-type Be stars. Regulus rotates about $86 \%$ critically (McAlister et al. 2005) and has a WD companion (Gies et al. 2008). Rappaport et al. (2009) traced out the past and future evolution of this system and found that the B star may evolve into an sdB star. In addition, Cracco et al. (2018) recently identified some supersoft X-ray sources with Be stars in the Magellanic Clouds. These sources are often intermittent and may be massive WDs occasionally igniting accreted matter, for example from a Be disc. Apparently, unlike in BeXRBs, the release of gravitational energy does not play a major role in such systems.

\section{2. sdO stars}

Two of the first Be stars initially suspected and later demonstrated to be orbited by a low-mass star strongly interacting with the Be disc were HR 2142 (Peters et al. 2016) and $\phi$ Per (Mourard et al. 2015). In UV spectra (mostly from IUE) with sufficient orbital phase coverage, spectral lines can be clearly seen with a much larger velocity swing than that of the B-type primary (Thaller et al. 1995). Numerous narrow Fe IV, V, and VI lines as well as the He II $\lambda 1640$ line convincingly show the similarity to spectra of sdO stars (Gies et al. 1998). A more indirect indicator of a hot companion can be a hot spot in the disc where helium emission lines form. Periodic shifts in radial velocity trace the secondary's orbit (Rivinius et al. 2004).

In stars with only a few scattered UV spectra, crosscorrelations of the observations with model spectra have been used to identify additional sdO companions and candidates (Wang et al. 2018). As explained in Sects. 2.2 and 2.3, this method is not very effective for broad-lined early-type Be stars, meaning many $\gamma$ Cas stars. The relatively low detection rate is probably also due to the low $\mathrm{S} / \mathrm{N}$ ratio of IUE spectra. The total number of Be stars with a detected or likely sdO companion is about 15 (Wang et al. 2018).

\subsection{Neutron stars and black holes}

Systems with neutron-star and black-hole companions (currently, only one Be system with a black hole seems to be known
Casares et al. 2014), for example BeXRBs, are omitted from the discussion, because, as outlined above, the X-ray properties of $\gamma$ Cas stars seem incompatible with those of BeXRBs, and there is no convincing evidence that the companions of $\gamma \mathrm{Cas}$ and $\pi$ Aqr are neutron stars or even black holes. However, the immediate progenitors of BeXRBs, namely Be stars with a helium-star companion have not yet been placed into a close perspective with the formation of Be stars; this is done in Sect. 5.

\section{Binary stellar evolution models}

Since stars, during their evolution, tend to increase their radii by large factors, most close binary systems will experience transfer of mass between the two stars. For the closest binaries, meaning for orbital periods typically below $\sim 10 \mathrm{~d}$, mass transfer starts while both stars undergo core hydrogen burning (Case A; Pols \& Marinus 1994; Pols 1994; Wellstein et al. 2001). In this case, the mass transfer is divided into three distinct phases: a thermal-timescale mass transfer (fast Case A), which is succeeded by a nuclear-timescale mass transfer phase, during which the mass ratio is inverted (slow Case A or Algol phase), followed by another thermal-timescale mass-transfer once the donor star ends core hydrogen burning (Case AB). In wider binary systems, the post-main-sequence expansion of the initially more massive star leads to thermal-timescale mass transfer, while the companion is generally still burning hydrogen (Case B). In both cases, the mass transfer may become unstable, with the likely consequence of a merger of both stars (de Mink et al. 2014). However, if a merger is avoided, the mass donor - the initially more massive star - loses almost its entire hydrogen-rich envelope due to mass transfer, while the mass gainer is accreting all, or only part of it. The ratio of the number of mergers and the number of stable mass-transfer systems, and the mass-transfer efficiency, are uncertain (Langer 2012).

Struve (1963) and Huang (1966) realised that the accretion of mass from a companion star can lead to an increase in the star's specific angular momentum, with the consequence that mass gainers may spin supersynchronously with regard to the orbital rotation. This effect is observationally well-documented for massive Algol systems (e.g., Howarth et al. 2015; Mahy et al. 2020), where spin-up to critical rotation is avoided through tidal spinorbit coupling (de Mink et al. 2013).

Only after Case AB or Case B mass transfer, if the mass transfer is not too inefficient, does one expect the spin-up process to drive the mass gainer towards critical rotation, since the orbits become wide enough to render tides negligible. It was shown analytically by Packet (1981), and later through detailed models by Petrovic et al. (2005), that a mass increase by only $10 \%$ can be sufficient to spin up a star to its critical rotation. The problem with this situation is that after Case AB or Case B mass transfer, the envelope mass of the donor becomes very small, to the extent that the donor is hotter than a main sequence star, and thus remains very faint in optical light. Furthermore, its remaining lifetime is much shorter than that of its spun-up companion. This means it will rapidly evolve into a compact object, which, in case a neutron star or black hole is formed, may lead to the disruption of the binary by the supernova explosion. As a consequence, most post-Case AB or Case B systems may not be recognised as such (de Mink et al. 2014).

In the following, it is assumed that the mass gainers of Case AB or Case B are in fact spun up in a way that they appear as $\mathrm{Oe} / \mathrm{Be}$ stars after the mass transfer. This idea is, of course, strongly supported by the large number of classical BeXRBs, which are explained as such post-Case $\mathrm{AB}$ or Case $\mathrm{B}$ binaries 
N. Langer et al.: $\gamma$ Cas stars: Normal Be stars with discs impacted by the wind of a helium-star companion?

Table 2. Key data of selected massive binary evolution models from Wellstein \& Langer (1999) and Wellstein et al. (2001).

\begin{tabular}{cccccccccccccc}
\hline \hline No. & $\begin{array}{c}M_{1, i} \\
M_{\odot}\end{array}$ & $\begin{array}{c}M_{2, i} \\
M_{\odot}\end{array}$ & $\begin{array}{c}P_{\mathrm{orb}, i} \\
\mathrm{~d}\end{array}$ & $\begin{array}{c}P_{\mathrm{He}+\mathrm{OB}} \\
\mathrm{d}\end{array}$ & $\begin{array}{c}M_{\mathrm{He}} \\
M_{\odot}\end{array}$ & $\begin{array}{c}M_{\mathrm{OB}} \\
M_{\odot}\end{array}$ & $\begin{array}{c}L_{\mathrm{He}} \\
10^{3} L_{\odot}\end{array}$ & $\begin{array}{c}L_{\mathrm{OB}} \\
10^{3} L_{\odot}\end{array}$ & $\begin{array}{c}T_{\mathrm{He}} \\
\mathrm{kK}\end{array}$ & $\begin{array}{c}T_{\mathrm{OB}} \\
\mathrm{kK}\end{array}$ & $\begin{array}{c}\log \dot{M}_{\mathrm{He}} \\
M_{\odot} \mathrm{yr}^{-1}\end{array}$ & $\begin{array}{c}v_{\mathrm{esc}, \mathrm{He}} \\
\mathrm{km} \mathrm{s}^{-1}\end{array}$ & $\begin{array}{c}L_{\text {wind,He }} \\
L_{\odot}\end{array}$ \\
\hline 1 & 12 & 8 & 2 & 189 & 1.1 & 18.5 & 0.436 & 69 & 48 & 32 & -9.71 & 1170 & 0.40 \\
2 & 10 & 8 & 3 & 61 & 1.8 & 16.1 & 2.7 & 29 & 57 & 32 & -8.63 & 1130 & 2.25 \\
3 & 12 & 8 & 6 & 72 & 2.4 & 17.5 & 6.2 & 35 & 65 & 33 & -8.14 & 1200 & 7.74 \\
4 & 16 & 13 & 3 & 64 & 2.6 & 25.3 & 6.9 & 120 & 74 & 35 & -8.08 & 1390 & 12.00 \\
5 & 16 & 15 & 9 & 107 & 3.6 & 26.7 & 16.6 & 126 & 80 & 38 & -7.56 & 1420 & 41.20 \\
6 & 25 & 19 & 4 & 30 & 5.3 & 35.9 & 35.0 & 238 & 98 & 43 & -7.12 & 1750 & 180.10 \\
\hline
\end{tabular}

Notes. Besides the initial binary parameters, i.e. the initial masses of the mass donor $\left(M_{1, i}\right)$ and the mass gainer $\left(M_{2, i}\right)$, and the initial orbital period $P_{\mathrm{orb}, i}$, we give parameters of the binary and its component stars at the time where the mass donor has a central helium mass fraction of 0.8 during core helium burning, i.e. the orbital period $P_{\mathrm{He}+\mathrm{OB}}$, both stellar masses during that stage, the corresponding luminosities and effective temperatures, and the expected stellar wind mass-loss rate, velocity and mechanical wind energy production rate, according to Vink (2017).

where the donor star evolved into a neutron star without breaking up the binary (Tauris \& van den Heuvel 2006). In these systems, the nature of the companion is revealed by the copious X-ray emission, which is produced when the neutron star crosses or approaches the Be disc in its tilted and/or elliptical orbit, which leads to mass accretion onto the neutron star. As seen in Sect. 4, there is also a smaller number of Be stars with known or suspected $\mathrm{BH}, \mathrm{WD}$ or sdO companions, which all fit into the postmass transfer scenario.

It is worth pointing out that rotating, non-magnetic stars can spin down as a result of stellar-wind mass loss (Langer 1998). For the most massive main sequence stars, which lose a significant fraction of their initial mass through a wind, this process may be efficient, and observational evidence for this exists in Galactic O stars (Markova et al. 2018). It also explains the fast but sub-critical rotation of the O stars in Galactic WR+Ostar binaries (Vanbeveren et al. 2018), in which the WR star was likely the mass donor in a mass transfer process (Petrovic et al. 2005). However, $\gamma$ Cas stars are Be/Oe stars that do not spin down. This is consistent with the expectation that the main sequence mass loss in Galactic stars is below $10 \%$ for stars with an initial mass below $28 M_{\odot}$ (Brott et al. 2011; Langer 2012).

\subsection{The case for $\gamma$ Cas stars as Be+helium-star binaries (BeHeBs)}

According to the above considerations, an $\mathrm{Oe} / \mathrm{Be}$ star, when it is formed as such in a binary system, has a helium star companion. The corresponding $\mathrm{Be}+$ helium-star binaries are below referred to as BeHeBs for short. While the helium star evolves faster than the Be star, the lifetime of this BeHeB stage - the helium burning timescale of the helium star - is long enough to expect that some of the observed Be binaries are in this stage (cf. Sect. 5.1.3).

This section features a discussion of the hypothesis that $\gamma$ Cas stars are BeHeBs, based on binary evolution models computed by Wellstein \& Langer (1999) and Wellstein et al. (2001). While these models do not include rotation, they assume conservative mass transfer, which implies that the mass increase is sufficient to spin up the mass gainer to critical rotation. Table 2 gives an overview of the initial parameters of these models, and those during the BeHeB stage. These were chosen in a way that the masses of the formed helium stars $\left(1.1-5.3 M_{\odot}\right)$ cover the plausible mass range of such objects in $\gamma$ Cas stars. That is, more massive helium stars would likely form optically-thick winds, which would make them easily identifiable as Wolf-Rayet stars (Langer 1989). And helium stars significantly below $1 M_{\odot}$ even require progenitors of initial mass so low that the mass gainer could not evolve into a B star of the earliest spectral type.

Figure 3 gives an overview of the evolution of both components of the binary models in the Hertzsprung-Russell diagram. The tracks of the pairs of stars start on the zero-age main sequence. While these evolutionary tracks otherwise show the typical pattern of Case A and B binary models (cf. Wellstein et al. 2001), the thick-drawn part of the lines focuses on the BeHeB stage, meaning on the time period during which the mass donor evolves through core helium burning.

As the mass gainers - the presumed later Be stars - hardly evolve during this time, the thick-drawn stretch of their evolutionary tracks is very short. For the mass donors, however, there is significant evolution. In any case, it is important to realise that the donors move fast along the horizontal parts of the evolutionary tracks. The thick dots on their tracks mark a central helium mass fraction of $Y_{\mathrm{c}}=0.8$, and core helium exhaustion is signified by the end of the thick-drawn part of the track. Therefore, the time-averaged properties of the helium stars are well represented by their properties at $Y_{\mathrm{c}}=0$.

It follows from Fig. 3 that the helium-star secondaries would be difficult to observe, in the optical and at longer wavelengths, next to the much brighter Be star (Götberg et al. 2018). However, with luminosities of 500 to $50000 L_{\odot}$, helium stars are still luminous stars, and as such they are expected to emit a radiation-driven wind. Observational evidence for this is found in the UV spectra of the rare so-called extreme helium stars (Jeffery \& Hamann 2010). While helium-star wind mass loss based on Hamann et al. (1982) is included in the presented binary evolution models, the present study uses the recent theoretical mass-loss rates by Vink (2017), which reproduce the empirical rates of Hamann et al. reasonably well, but also provide a smooth transition to the mass-loss properties of the more massive Wolf-Rayet stars. As the total amount of mass lost during core helium burning is mostly very small, this does not introduce any significant inconsistency.

The models provide guidance in answering the question of whether the presence of a wind emanating from the helium star could give rise to an observable X-ray signal in BeHeBs. As the helium stars are compact, and their winds fast, the models lead to the expectation of X-ray emission from two potential interaction regions. The first candidate zone is where the wind of the He star encounters the disc of the Be star, and the second one resides where the He-star wind meets the - also present - ordinary radiation-driven wind of the Be star. The following subsections examine these two cases. 


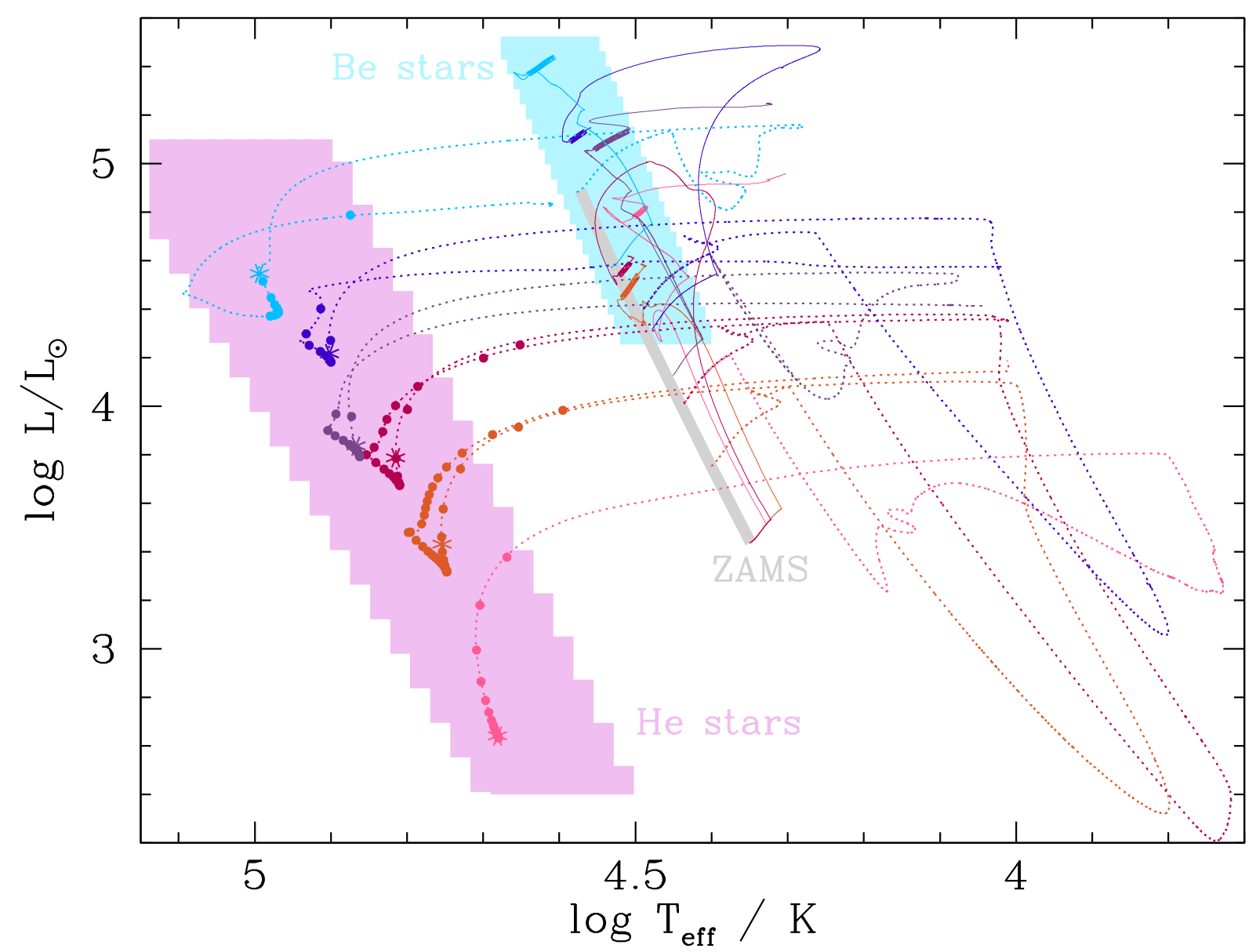

Fig. 3. Evolutionary tracks of mass donors (dotted lines) and mass gainers (solid lines) of the analysed six binary models in the HertzsprungRussell diagram. Pairs of tracks with the same colour belong to the same binary system. The thick grey line marks the zero-age main sequence for the initial mass range of our models (i.e. from 8 to $25 M_{\odot}$ ). The thick solid drawn parts of the mass gainers' tracks marks the phase during which the companion is a He star (starting from a core helium mass fraction of $Y_{\mathrm{c}}=0.95$ during core helium burning). Corresponding area in HR diagram is coloured light blue and labelled "Be stars". On the tracks of the mass donors, dots are placed every $10^{5} \mathrm{yr}$ during core helium burning, and a star symbol marks the time at which their core helium mass fraction is $Y_{\mathrm{c}}=0.80$. The tracks end during the phase of shell helium burning with a small remaining lifetime of the He stars, except for the System No. 1, which ends at $Y_{\mathrm{c}}=0.7$. Area in the HR diagram in which He star models spend most of their lifetime is coloured in pink and labelled "He stars". Tracks correspond to binary models Nos. 1 to 6 (Table 2) in increasing order of helium-star luminosity (as marked by the star symbols).

\subsubsection{Interaction between He-star wind and Be disc}

If the He star had no wind, the Be disc might well extend to the He star companion or even engulf it. This is so by analogy to the BeXRBs, where a neutron star, meaning, the descendant of a helium star in a BeHeB, emits X-rays when it crosses the equatorial plane of the Be star. Since He stars possess a strong wind, they will blow a cavity into the Be disc, whose size may be determined by the balance of the wind ram pressure and the thermal and turbulent pressure of the gas in the Be disc. The cavity may be elongated in the direction of the orbital motion, and its vertical size will depend on the thickness and vertical structure of the Be disc. Truncation by the companion of the disc (Panoglou et al. 2018) could lead to still other geometries. Some fraction of the He-star wind could escape without interacting with the disc.

In any case, the interaction shock front would likely have a complex three-dimensional structure, and may develop turbulence and magnetic fields, which would all affect the emission of energetic photons. In a first simple step, the next paragraphs attempt to derive upper limits on the X-ray luminosity and the photon temperature from predictions of stellarevolution and radiation-driven-wind physics.
Figure 4 illustrates the time dependence of the helium star's mechanical wind luminosity $L_{\text {wind,He }}=\frac{1}{2} \dot{M}_{\mathrm{He}} v_{\text {wind,He }}^{2}$ for the six evolutionary models in Table 2 . Here, $\dot{M}_{\mathrm{He}}$ is the mass-loss rate predicted by Vink (2017), and $v_{\text {wind,He }}$ is the terminal wind velocity, for which Vink showed that it exceeds the escape speed of the helium stars by about a factor of three. It is, therefore, assumed here that $v_{\text {wind,He }}=3 \sqrt{2 G M_{\mathrm{He}} / R_{\mathrm{He}}}$.

Figure 4 also provides an upper limit to the X-ray luminosity produced by the wind-disc interaction, because only a fraction of the kinetic energy can be converted to X-rays. As the figure shows, the wind kinetic-energy fluxes are of the order of a few hundred $L_{\odot}$ for the massive helium stars $\left(M \simeq 5 M_{\odot}\right)$ down to fractions of $L_{\odot}$ at lower masses $\left(M \simeq 1 M_{\odot}\right)$. These numbers should only be taken as order-of-magnitude indicators, since, in his pioneering study, Vink (2017) adopted a fixed effective temperature of $50000 \mathrm{~K}\left(\log T_{\text {eff }} \simeq 4.7\right.$; cf. Fig. 3$)$ while the temperature dependence of these winds is not yet well understood. Clearly, even lower wind luminosities will occur in systems with masses below the range considered here. However, as potentially observable effects will become correspondingly weaker, they are not considered here. More massive systems, on the other hand, might contain $\mathrm{O}$ stars whose strong winds would - at least at 


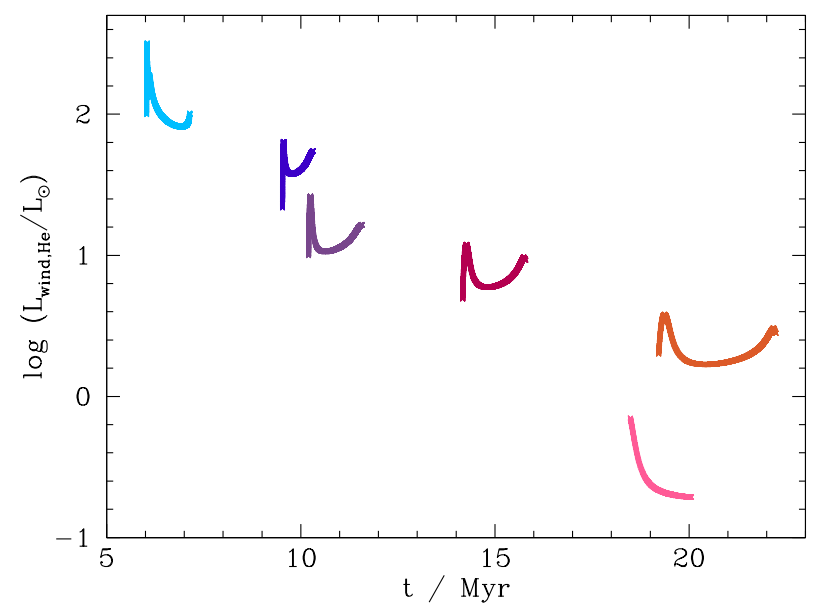

Fig. 4. Time evolution of mechanical luminosity of the donor star's wind during its helium-star stage, for the six binary models during core helium burning of the donor star. The colour coding is the same as in Fig. 3, and the tracks belong to binary models Nos. 1 to 6 (Table 2) in increasing order of their wind luminosity.

Galactic metallicities - spin down the stars, to an extent that they would not be $\mathrm{Oe} / \mathrm{Be}$ stars for long. Potentially, they would also destroy any circumstellar disc.

As for the X-ray luminosity, the given models only place an upper limit on the temperature of the hot gas, which is produced by the shock front where the He-star wind hits the $\mathrm{Be}$ disc. With escape speeds of the helium stars in the range of $1100-1800 \mathrm{~km} \mathrm{~s}^{-1}$, the terminal wind speeds of the He star are of the order of $3000-5000 \mathrm{~km} \mathrm{~s}^{-1}$ (see above). For an adiabatic shock, these numbers translate to temperatures of about $5 \times 10^{8} \mathrm{~K}$ to $15 \times 10^{8} \mathrm{~K}$, or 50 to $130 \mathrm{keV}$, assuming $T=$ $m_{\mathrm{p}} v_{\text {wind,He }}^{2} /(2 k)$, where $m_{\mathrm{p}}$ is the mass of the proton. On the other hand, at sufficiently high densities, the shock may be nonadiabatic, so the achieved temperature can be much smaller. Hydrodynamic instabilities, clumping, or entrainment of cold gas may also lead to smaller temperatures. However, this is challenging to estimate quantitatively, and beyond the scope of the present work.

\subsubsection{Interaction between $\mathrm{He}$-star wind and Be-star wind}

As mentioned above, a fraction $f_{1}<1$ of the He-star wind may be able to escape without interacting with the Be disc. Part of this matter would, however, collide with the ordinary Be-star wind, so that only a fraction $f_{1} * f_{2}$ of the He-star wind leaves the system without any interaction at all. Here, $f_{2}<1$ designates the fraction of the He-star wind not hitting the Be disc that also escapes collision with the Be-star wind.

A key parameter determining the interaction fraction and also the X-ray production efficiency of colliding wind systems is the wind momentum ratio $\eta=\dot{M}_{\mathrm{He}} v_{\mathrm{He}} / \dot{M}_{\mathrm{OB}} v_{\mathrm{OB}}$, where $\dot{M}$ and $v$ denote the mass-loss rates and terminal wind velocities of both stars, respectively. The interaction fraction and the X-ray production efficiency are largest for $\eta=1$ (Pittard \& Dawson 2018). Figure 5 illustrates the time dependence of $\eta$ for the selected binary-model sequences during the stage of core $\mathrm{He}$ burning of the helium star. The underlying mass-loss rates and terminal wind velocities are those proposed by Krtička (2014) for ordinary B main-sequence stars. As the terminal wind speeds of Krtička's wind models are roughly three times the corresponding escape speed from the star, the escape speed of the models in Table 2 was multiplied by a factor of three to compute their terminal wind speeds. This neglects the possibility that in very

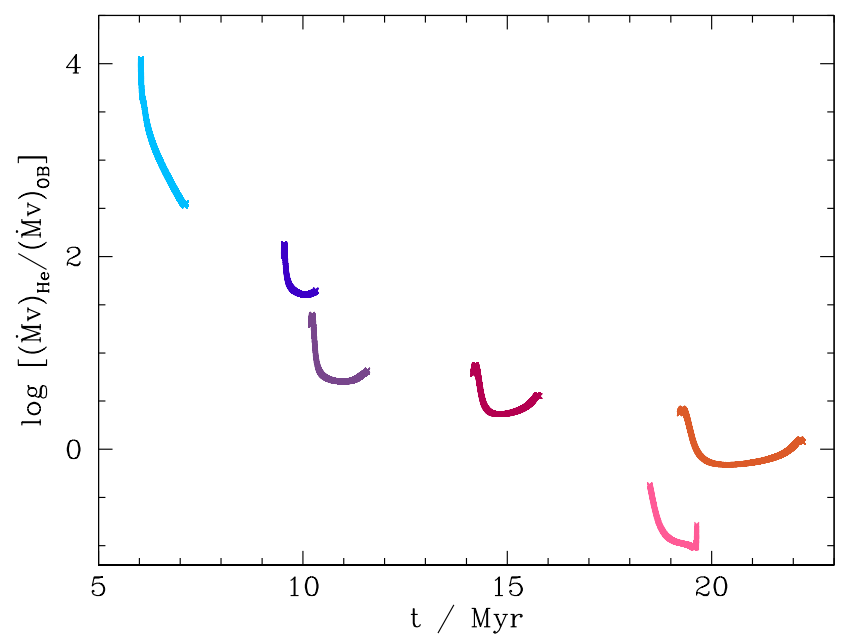

Fig. 5. Time evolution of ratio $\eta$ of wind momentum of the donor star to that of the mass gainer for six binary models (see Table 2) during core helium burning of the donor star. The colour coding is the same as in Fig. 3.

close systems, or for values of $\eta$ far from unity, one or both winds might not quite have attained their terminal speeds when reaching the interaction point.

Figure 5 demonstrates that, in the considered binary models, quite diverse situations may prevail. In some systems (typically the more massive ones), the He-star wind momentum is larger by more than an order of magnitude, and in some other systems (typically the less massive ones) the B-star wind is stronger by a similar factor, and in others, the wind momentum ratio is close to unity. This occurs because both the wind velocities and the mass-loss rates of $\mathrm{He}$ - and B-stars are not too different.

Similar to the wind-disc interaction, it is difficult to provide firm predictions for the $\mathrm{X}$-ray emission produced by the wind-wind interaction. The upper limits to the X-ray flux and plasma temperature are similar to those of the wind-disc interaction, since the wind velocities and mass-loss rates are also similar. On the other hand, the conditions in the wind-wind interaction region would be different from those in the wind-disc case, since, for example, the matter density in the Be disc would be larger than that in the Be-star wind. It is therefore possible that X-ray emission will be composed of more than one discrete component.

Observations of colliding-wind binaries show that the $\mathrm{X}$-ray flux in massive He-star+-O-star binaries can reach about $100 L_{\odot}$ and temperatures up to $100 \mathrm{MK}$ (Gagné et al. 2012). The mass-loss rates in these systems are several orders of magnitude above those in BeHeBs, but the wind velocities are comparable. Pittard \& Dawson (2018) find from hydrodynamical simulations that the expected X-ray emission decreases roughly linearly with the weaker-to-stronger wind-momentum ratio. Moreover, in BeHeBs, the wind-wind interaction is restricted to higher latitudes, as the equatorial regime is blocked by the Be disc. Nevertheless, while there are several factors that may reduce the X-ray emission, a detectable X-ray flux from the wind-wind interaction is not excluded, especially not for $\mathrm{BeHeBs}$ with a wind momentum ratio near unity, which is achieved by the majority of the models considered (Table 2).

\subsubsection{The expected number of BeHeBs}

The $\mathrm{BeHeB}$ phase is a short intermediate evolutionary phase of massive binary systems, for which direct observational evidence 
is still lacking. This phase is defined by the core helium-burning stage of the mass donor. It is often disregarded in comparison with observations, because its duration is mostly shorter than that of the foregoing Algol phase (if any), but also shorter than the subsequent $\mathrm{BeXRB}$ or $\mathrm{Be}+\mathrm{WD}$ phase.

The binary evolution models of Wellstein et al. (2001) can give an estimate of the number of $\mathrm{BeHeBs}$ relative to $\mathrm{BeXRB}$ or Be+WD systems. The stripped core helium-burning companions to B-star mass gainers are very hot $\left(T_{\text {eff }} \gtrsim 50 \mathrm{kK}\right)$ and subluminous (cf. Fig. 3), leading to no realistically observable signal in the optical regime which is dominated by the B star. The lifetime of the faint, hot helium star is the nuclear timescale of core helium burning, which is of the order a few Myr for helium stars in the mass range 1.6-5 $M_{\odot}$ (Woosley 2019). Compared to the hydrogen-burning lifetimes of the rejuvenated B-star mass gainers of 10 to $30 \mathrm{Myr}$, this is about $10 \%$ or less. Therefore, among the Be stars that have emerged from this binary evolution channel, a comparable fraction, meaning up to $10 \%$, could have a helium-star companion.

For an accurate prediction of the number of $\gamma$ Cas binaries expected from the ansatz pursued above, a population-synthesis study is probably required. Clearly, the estimate of $\sim 10 \%$ for the number ratio of $\mathrm{BeHeBs}$ to $\mathrm{Be}$ binaries with compact companions (BeXRBs and Be+WD systems) can serve as an upper limit. However, direct empirical comparisons will suffer from a strong observational bias. $\gamma$ Cas binaries would be identified on account of their X-ray properties, while only $\gamma$ Cas binaries with sufficiently massive helium-star companions are predicted to have detectable X-ray fluxes. The latter subpopulation may roughly consist of those systems in which the helium stars end their evolution as neutron stars. The expected fraction of observed $\gamma$ Cas binaries would be $10 \%$ of the BeXRBs, multiplied by the luminosity bias factor, and divided by the break-up fraction, $f_{\text {breakup }}$ of Be binaries at neutron-star formation in a supernova explosion. Both factors are quite uncertain, but a fraction of about $10 \%$ of all BeXRBs (i.e. of all progenitor systems not disrupted by a supernova explosion) does not seem impossible.

\subsection{Comparison with observations}

As seen above, some fractions of the Be-star binaries (the $\mathrm{BeHeBs}$ ) are expected to contain a core helium-burning star. The He star is not likely to be readily observable, as it is bolometrically much dimmer than the B star. Because the He star is much hotter than the Be star, the contrast problem is lowest in the UV. The previous section considered corresponding binary-evolution and stellar-wind models, with the idea in mind that the presence of a helium star may give rise to observable X-ray emission. In the following, we discuss to what extent the $\gamma$ Cas stars and their peculiar X-ray properties (cf. Sect. 2.5) might correspond to the BeHeBs. To this effect, the recent compilation of $\gamma$ Cas stars by Nazé \& Motch (2018) is used, from which the quantities in Table 1 were drawn.

Firstly, it should be noted that, in BeHeBs, there may be other sources of X-rays than those that are induced by the fast wind of the helium star. In particular, the helium star itself can be so hot that it emits X-rays. For example, for $T_{\text {eff }}=100000 \mathrm{~K}$, the Planck function peaks at $0.3 \mathrm{keV}$. As this is the hottest temperature expected for BeHeBs, it follows that only very soft X-rays can be produced in this way. This holds similarly for the thermal emission of hot pre-white dwarfs, as well as for accreting white dwarfs (cf. Cracco et al. 2018). As the X-rays measured in $\gamma$ Cas stars are much harder (cf. Sect. 2), they are unlikely to be produced in stellar photospheres.

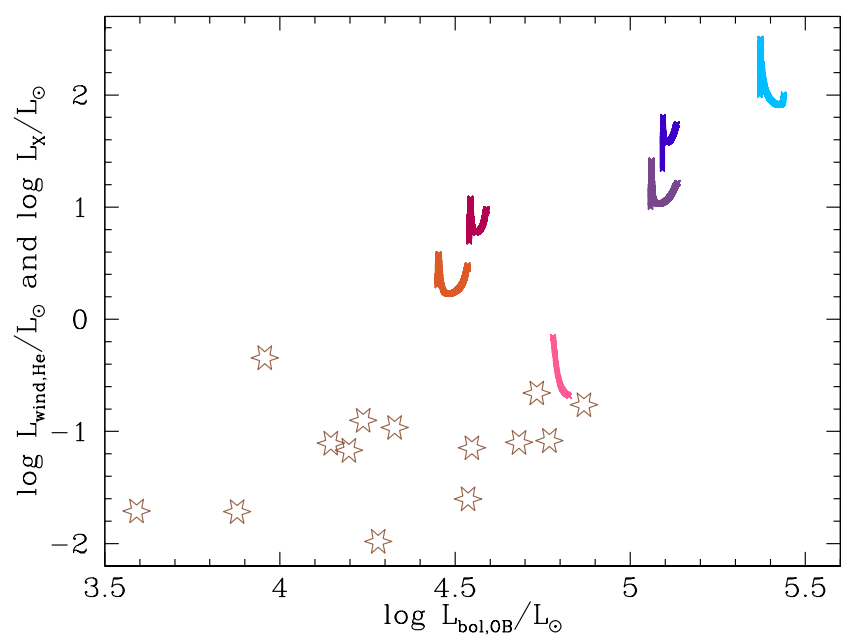

Fig. 6. Mechanical luminosities of donor star winds versus the mass gainer's bolometric luminosity for six binary models (cf. Table 2), during core helium of the donor star. The colour coding is the same as in Fig. 3, and the tracks belong to binary models Nos. 1 to 6 (Table 2) in increasing order of their wind luminosity. Also plotted are X-ray luminosities of $\gamma$ Cas stars versus their bolometric luminosities, according to Nazé \& Motch (2018, see also Table 1).

Secondly, in Fig. 6, the mechanical luminosities of the helium-star winds as a function of the OB star's bolometric luminosity for the six model systems (Table 2) are plotted together with the observed X-ray luminosities of the $\gamma$ Cas stars and their respective bolometric luminosities. Here, it should be stressed that both predicted (cf., Sects. 5.1.1 and 5.1.2) and observed quantities (Table 1) have large uncertainties. In particular, the bolometric luminosities of the Be stars could be wrong a factor of $2-3$, since their luminosity classes are only adopted, individual extinction corrections have not been determined, and the rapid rotation of the Be stars leads to an anisotropy of the photon emission (von Zeipel 1924), which is unaccounted for.

There is some overlap in the areas populated by models and observations in Fig. 6. Also, the bolometric luminosities of both samples span about a factor of 20 , and the ordinate range of models and observations span 2-3 dex. However, there are considerable offsets in both coordinates between the two datasets, namely by about a factor of 3 in $L_{\text {bol,OB }}$, and a factor of 100 comparing $L_{\text {wind,He }}$ and $L_{X}$.

Specifically considering the ordinate of Fig. 6, the difference may arise because the conversion of mechanical wind energy to $\mathrm{X}$-rays in $\gamma$ Cas binaries is an inefficient process. In fact, this generally seems to be the case for colliding-wind binaries as discussed above, in particular when the wind momentum ratio is far from unity. The conversion efficiency in the wind-disc interaction case is less clear, owing to the lack of comparable cases in other systems. However, we can conclude that the mechanical wind energy of the helium stars is sufficient to account for the observed energy in X-rays in $\gamma$ Cas stars.

The range in OB-star bolometric luminosities (the abscissa in Fig. 6) should be more directly comparable, in spite of the caveats mentioned above. In $L_{\mathrm{bol}, \mathrm{OB}}$, the overlap between models and observations is larger, but the models are generally more luminous. One reason for the difference is the inclusion of a fairly massive model (No. 6 in Table 1), mostly for illustrative purposes, as this may correspond better to $\mathrm{WR}+\mathrm{O}$-star binaries instead of BeHeBs. Certainly, at $36 M_{\odot}$ the mass gainer in this model becomes so massive that its wind spins it down quickly (Brott et al. 2011), so that its lifetime as an Oe star would be 
very short. However, it is also important to consider that the models of Wellstein et al. (2001) are mass-conserving, which means that the entire mass lost from the donor is accreted onto the mass gainer. Recent evidence shows, however, that mass transfer in massive close binaries may well be non-conservative on average (de Mink et al. 2007; Langer 2012). Because nonconservative evolution does not lead to a different evolution for the donor stars, the He-star properties of Wellstein et al. (2001) would remain about the same in the non-conservative case. However, the mass gainers, which are mostly in the late O-star regime in the models analysed above, would be significantly less massive, and thus less luminous. That is, the tracks of the models shown in Fig. 6 would move to the left at constant ordinate. In extreme cases, the mass gainer's mass would just be about half of what it becomes in the conservative model, thereby decreasing $\log L / L_{\odot}$ by about 0.9 dex.

It seems unlikely that models and data could be brought into agreement by considering binary models with smaller initial masses. As seen in Fig. 6, models and data might overlap well if the downward trend of wind luminosity with bolometric luminosity continued. However, if the observed sample of $\gamma \mathrm{Cas}$ stars is merely the peak of a distribution that extends to lower $\mathrm{X}$-ray luminosities, the known $\gamma$ Cas stars should correspond to the most luminous models that predict the $\gamma$ Cas phenomenon. This range is obviously covered by the chosen theoretical tracks.

In summary, if $\gamma$ Cas stars are binaries with core heliumburning helium stars, two suggestions arise from Fig. 6. Firstly, the mass-transfer efficiency during the preceding mass-transfer phases was about 0.5 (since the model tracks would have to be shifted to the left by about 0.4 dex to match the data), and secondly, about $1 \%$ of the wind luminosity would be converted into X-rays.

Figure 7 compares the (mechanical) He-star wind luminosities and the $5 \mathrm{keV} / 1 \mathrm{keV}$ flux ratio using the black-body approximation and the adiabatic post-shock temperature of the winds with empirical data of $\gamma$ Cas stars from Nazé \& Motch (2018). Taken at face value, the X-ray luminosities as well as the adiabatic flux ratios derived from the models (Table 2) are much too high compared to the observed X-ray luminosity and hardness ratio. However, as discussed above, only about $1 \%$ of the mechanical wind luminosity needs to be converted to X-rays, thereby drastically reducing the apparent mismatch. At the same time, the flux ratio is predicted one order of magnitude too high, which means that the temperature needs to come down from $\sim 500 \mathrm{MK}(\sim 40 \mathrm{keV})$ to $\sim 15 \mathrm{MK}(\sim 1.3 \mathrm{keV})$. Only detailed modelling can show whether the X-ray properties can be brought into agreement with the observations.

The indications of line emission associated with the companion stars of $\gamma$ Cas (Miroshnichenko et al. 2002) and $\pi$ Aqr (Bjorkman et al. 2002) also support the notion of an interaction between the companion stars and the discs of the Be stars, although it is not clear whether this interaction is radiative, gravitational, or both. The asymmetric structure in the disc of BZ Cru (Stee et al. 2013) may have the same origin.

The BeHeB model also reproduces other observed properties of the $\gamma$ Cas stars. (i) Any interaction between He-star wind and Be disc will lead to a correlation between X-ray and optical continuum, as well as $\mathrm{H} \alpha$ line-emission flux. (ii) An interaction between He-star wind and Be disc will also often place the X-ray-emitting region closer to the B star than accretingcompanion models would. (iii) Injection of new matter into the disc can easily lead to increased line-of-sight column densities of X-ray-attenuating matter. (iv) Collision of the He-star wind with a Be-star wind strongly structured by co-rotating interac-

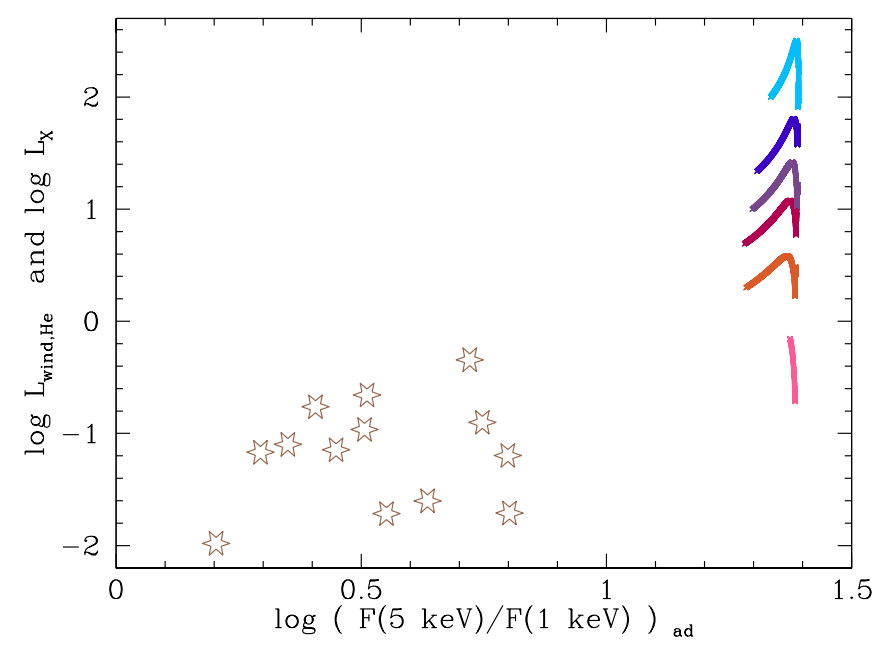

Fig. 7. Mechanical luminosities of donor-star winds versus $5 \mathrm{keV} / 1 \mathrm{keV}$ flux ratio assuming adiabatic shocks for the six binary models (cf. Table 2), during core helium burning of the donor star. The colour coding is the same as in Fig. 3. Also plotted (as stars) are observed X-ray luminosities and $5 \mathrm{keV} / 1 \mathrm{keV}$ flux ratios of $\gamma$ Cas stars versus their bolometric luminosities, according to Nazé \& Motch (2018) (cf. Table 1).

tion regions and/or with an azimuthally inhomogeneous Be disc, often fed by discrete stellar mass-loss events, may lead to variable X-ray flux on a broad range of time scales. Furthermore, the $\mathrm{X}$-ray emission that may arise from two distinct regions in the $\mathrm{BeHeB}$ model may well relate to the multi-temperature nature of the observed X-ray continuum in some $\gamma$ Cas stars (Sect. 2). A similar differentiation was already proposed by Hamaguchi et al. (2016).

\subsection{Further model predictions}

As discussed in Sect. 3.3, the supernova explosions that ultimately transform $\mathrm{BeHeBs}$ with massive helium-star components into BeXRBs lead to an increase in orbital eccentricity. By contrast, in the progenitors of BeXRBs, namely the BeHeBs, the previous mass-transfer evolution should reduce any earlier eccentricity to zero, and align the Be spin and the orbital angularmomentum vector. Therefore, the orbit of the helium star around the Be stars is expected to be circular and coplanar with the Be disc. Instead of being strongly orbitally modulated, as in BeXRBs (Okazaki \& Negueruela 2001), the X-ray production in $\mathrm{BeHeB}$ should thus be more continuous (but variable due to mass-loss events from the Be star). The truncation by the companion of the disc (Okazaki \& Negueruela 2001) would place the locus of formation of the X-rays slightly closer to the B star than to the helium star.

Other predictions resulting from the given ansatz are that $\gamma$ Cas stars might have rather massive helium-star companions. For most of the $\gamma$ Cas stars, it is not known whether they are binaries, let alone the masses of any companions. However, Nemravová et al. (2012) proposed that the secondary in $\gamma$ Cas is a helium star with a mass of about one $1 M_{\odot}$, and $\pi$ Aqr seems to have a companion mass that does not fit a white dwarf or a neutron star (2.2-4.5 $M_{\odot}$ Bjorkman et al. 2002). Nazé et al. (2017) suggested that the companion may be a main sequence star. However, this would not explain the Be nature of the primary, nor the level of the observed X-ray emission. Furthermore, as $\pi$ Aqr would be a wide pre-interaction binary in this case, a circular orbit would be very unlikely. By contrast, the masses 
of many other sdO stars reported for Be stars are far below one solar mass, which may be a challenge, as explained above.

The He-star wind may blow a significant cavity or even a hole into the Be disc. While model predictions of this are beyond the scope of this study, it is noteworthy that asymmetries seem to have been observed in some cases, for instance in BZ Cru (Sect. 2.4). Furthermore, the hot helium star can locally change the ionisation structure of the disc, leading to periodic orbital modulations as already observed in some Be binaries (Rivinius et al. 2004). Direct detection of the hot helium stars would best be attempted by orbital-phase-resolved UV spectroscopy (cf. Peters et al. 2013).

\section{Summary and conclusions}

Occam's razor offers the insight that the credibility of a proposed solution to a problem increases with the simplicity of the solution, where simplicity is often understood as the usage of established knowledge modules. While the existence and effects of companion stars can be addressed observationally (albeit only very tediously for any given individual Be system), the two magnetic fields of the magnetic model proposed for $\gamma$ Cas stars cannot by construction. Moreover, helium stars are known to exist, whereas magnetic fields caused by subsurface convection or MRI are still awaiting observational confirmation, even in objects they were originally designed for. On this ground, Occam would advise to first exhaust the explanatory power of binary models.

Previous binary models tried to explain the defining X-ray properties of $\gamma$ Cas stars in terms of accretion to white dwarfs or neutron stars. However, they are struggling in various ways to reproduce the observations fully. A generic objection apparently fuelled by the available observations could be that the release of gravitational energy that powers such systems can only take place close to these compact companions, meaning far away from the B star. Therefore, the present study has explored a different type of companion, namely the short-lived phase of B stars with a helium-star companion, $\mathrm{BeHeBs,} \mathrm{which} \mathrm{are} \mathrm{the} \mathrm{progeni-}$ tors of $\mathrm{BeXRBs}, \mathrm{Be}+\mathrm{WD}$ or $\mathrm{Be}+\mathrm{sdO}$ systems, depending on the mass of the helium star.

The collision in BeHeBs of a fast stellar wind from a companion with the $\mathrm{Be}$ disc and/or the $\mathrm{Be}$ wind as a different concept has a well-proven analogue in the colliding winds of the two components of massive binary systems. The discovery of BHeB stars, meaning, "normal" B stars with a helium-star companion but without circumstellar disc and without $\gamma$ Cas-like $\mathrm{X}$-ray properties, would favour the wind-disc collision part of the BeHeB model. A specific variant of this idea, namely the interaction of a WD wind with the Be disc, was first proposed by Hamaguchi et al. (2016). Because the X-rays do not have to be generated in the immediate vicinity of the companion, prospects are improved so much that detailed modelling can achieve good agreement with a wide range of observations. Closer to the B star and its mass-loss activity, the door is much wider open towards reproducing long-term but only little delayed correlations between variations in X-rays, optical flux, and UV spectral lines. In particular, outbursts may well supply the variable amounts of line-of-sight matter to explain the observed intermittent attenuations of the soft X-ray flux.

If $\gamma$ Cas stars do have a companion with such effects, it is clear that these stars must be of relatively low mass and low optical luminosity. With the additional restriction to stars with a strong wind, only helium stars and WDs remain as candidates. The helium-star wind model has the potential to place the hard- ness and flux of thermal X-rays from $\gamma$ Cas stars in the observed domains. More $\gamma$ Cas stars should be carefully screened for hot subluminous companions. The most conclusive results can be expected from UV spectroscopy.

There is no observational evidence of systematic differences other than the X-ray properties between $\gamma$ Cas stars and the general population of classical Be stars of the same spectral type. This agrees with our model, which is not dependent on any special assumptions about the Be stars themselves other than their rapid rotation. A full reunification of $\gamma \mathrm{Cas}$ and classical Be stars can be expected from a spectroscopic study of a representative $\gamma$ Cas star like that performed by Walker et al. (2005) for $\zeta$ Oph. First positive diagnoses of multimode NRP already exist for $\gamma$ Cas (Sect. 2.2) and $\pi$ Aqr (Sect. 2.3). Such work may also lead to coarse predictions of mass-loss outbursts of the B star (Rivinius et al. 1998; Baade et al. 2018) and thereby facilitate parallel optical and X-ray spectroscopy when the wind of the helium star interacts with ejecta from the B star. A first estimate of the relative $\mathrm{X}$-ray contributions by interactions between the He-star wind and the Be disc or the Be wind, respectively, may also result. Combined spectroscopy and photometry may provide valuable diagnostics of disc regions not well-probed by other observations, which are mostly biased towards the denser parts.

Because it is difficult to find Be stars that do not pulsate, it may be feasible (it certainly is attractive) to search for any statistical differences between the pulsation properties of bona fide single Be stars and Be stars with different kinds of companion (neutron stars, WDs, sdO stars, main-sequence stars): Can such a first crude step towards asteroseismology of Be stars distinguish formation channels of Be stars?

Furthermore, BeHeB stars should be valuable academies of the short-lived helium stars and their role in the evolution of massive binaries. An identification of $\gamma$ Cas stars with these objects would provide the missing link between the unevolved main-sequence binaries and Be binaries with compact companions.

Acknowledgements. We thank the referee, Dr. Georges Meynet, for useful comments and suggestions. J.B. acknowledges support from the FWO Odysseus program under project G0F8H6N. This publication makes use of data products from the Wide-field Infrared Survey Explorer (WISE), which is a joint project of the University of California, Los Angeles, and the Jet Propulsion Laboratory/California Institute of Technology, funded by the National Aeronautics and Space Administration. This research has made use of the SIMBAD database (Wenger et al. 2000) and the VizieR catalog access tool (Ochsenbein et al. 2000), both operated at CDS, Strasbourg, France. This research has made use of NASA's Astrophysics Data System (ADS).

\section{References}

Baade, D., Rivinius, T., Pigulski, A., et al. 2017, in Second BRITE-Constellation Science Conf.: Small Satellites - Big Science, eds. K. Zwintz, \& E. Poretti, Proc. Polish Astron. Soc., 5, 196

Baade, D., Rivinius, T., Pigulski, A., et al. 2018, in 3rd BRITE Science Conference, eds. G. A. Wade, D. Baade, J. A. Guzik, \& R. Smolec, 8, 69 Bernhard, K., Otero, S., Hümmerich, S., et al. 2018, MNRAS, 479, 2909

Bjorkman, K. S., Miroshnichenko, A. S., McDavid, D., \& Pogrosheva, T. M. 2002, ApJ, 573, 812

Bodensteiner, J., Baade, D., Greiner, J., \& Langer, N. 2018, A\&A, 618, A110 Boubert, D., \& Evans, N. W. 2018, MNRAS, 477, 5261

Brott, I., de Mink, S. E., Cantiello, M., et al. 2011, A\&A, 530, A115

Cantiello, M., \& Braithwaite, J. 2011, A\&A, 534, A140

Casares, J., Negueruela, I., Ribó, M., et al. 2014, Nature, 505, 378

Cohen, D. H. 2000, in IAU Colloq. 175: The Be Phenomenon in Early-Type Stars, eds. M. A. Smith, H. F. Henrichs, \& J. Fabregat, ASP Conf. Ser., 214, 156

Cohen, D. H., Cassinelli, J. P., \& MacFarlane, J. J. 1997, ApJ, 487, 867 
N. Langer et al.: $\gamma$ Cas stars: Normal Be stars with discs impacted by the wind of a helium-star companion?

Cracco, V., Orio, M., Ciroi, S., et al. 2018, ApJ, 862, 167

Cranmer, S. R., \& Owocki, S. P. 1996, ApJ, 462, 469

del Valle, M. V., \& Romero, G. E. 2012, A\&A, 543, A56

de Mink, S. E., Pols, O. R., \& Hilditch, R. W. 2007, A\&A, 467, 1181

de Mink, S. E., Langer, N., Izzard, R. G., Sana, H., \& de Koter, A. 2013, ApJ, 764, 166

de Mink, S. E., Sana, H., Langer, N., Izzard, R. G., \& Schneider, F. R. N. 2014, ApJ, 782, 7

Gagné, M., Fehon, G., Savoy, M. R., et al. 2012, ASP Conf. Ser., 465, 301

Ghoreyshi, M. R., Carciofi, A. C., Rímulo, L. R., et al. 2018, MNRAS, 479, 2214

Gies, D. R., Bagnuolo, Jr., W. G., Ferrara, E. C., et al. 1998, ApJ, 493, 440

Gies, D. R., Bagnuolo, Jr., W. G., Baines, E. K., et al. 2007, ApJ, 654, 527

Gies, D. R., Dieterich, S., Richardson, N. D., et al. 2008, ApJ, 682, L117

Götberg, Y., de Mink, S. E., Groh, J. H., et al. 2018, A\&A, 615, A78

Grady, C. A., Bjorkman, K. S., Snow, T. P., et al. 1989, ApJ, 339, 403

Granada, A., Ekström, S., Georgy, C., et al. 2013, A\&A, 553, A25

Güdel, M., \& Nazé, Y. 2009, A\&ARv, 17, 309

Haberl, F. 1995, A\&A, 296, 685

Haberl, F., \& Sturm, R. 2016, A\&A, 586, A81

Hamaguchi, K., Oskinova, L., Russell, C. M. P., et al. 2016, ApJ, 832, 140

Hamann, W.-R., Schoenberner, D., \& Heber, U. 1982, A\&A, 116, 273

Harmanec, P. 2002, ASP Conf. Ser., 279, 221

Harmanec, P., Habuda, P., Štefl, S., et al. 2000, A\&A, 364, L85

Henrichs, H. F., Hammerschlag-Hensberge, G., Howarth, I. D., \& Barr, P. 1983, ApJ, 268, 807

Henry, G. W., \& Smith, M. A. 2012, ApJ, 760, 10

Hoffleit, D., \& Jaschek, C. 1991, The Bright Star Catalogue (New Haven: Yale University Observatory)

Horaguchi, T., Kogure, T., Hirata, R., et al. 1994, PASJ, 46, 9

Howarth, I. D., \& Prinja, R. K. 1989, ApJS, 69, 527

Howarth, I. D., Dufton, P. L., Dunstall, P. R., et al. 2015, A\&A, 582, A73

Huang, S.-S. 1966, ARA\&A, 4, 35

Jahoda, K., Swank, J. H., Giles, A. B., et al. 1996, Proc. SPIE, 2808, 59

Jeffery, C. S., \& Hamann, W. R. 2010, MNRAS, 404, 1698

Jernigan, J. G. 1976, IAU Circ., 2900, 1

Kambe, E., Hirata, R., Ando, H., et al. 1997, ApJ, 481, 406

Kee, N. D., Owocki, S., \& Kuiper, R. 2018, MNRAS, 474, 847

Krtička, J. 2014, A\&A, 564, A70

Kř́̌ž, S., \& Harmanec, P. 1975, Bull. Astron. Inst. Czechoslov., 26, 65

Labadie-Bartz, J., Chojnowski, S. D., Whelan, D. G., et al. 2018, AJ, 155, 53

Langer, N. 1989, A\&A, 210, 93

Langer, N. 1998, A\&A, 329, 551

Langer, N. 2012, ARA\&A, 50, 107

Lee, U., Osaki, Y., \& Saio, H. 1991, MNRAS, 250, 432

Lopes de Oliveira, R., Smith, M. A., \& Motch, C. 2010, A\&A, 512, A22

Mahy, L., Almeida, L. A., Sana, H., et al. 2020, A\&A, in press https://doi org/10.1051/0004-6361/201936152

Markova, N., Puls, J., \& Langer, N. 2018, A\&A, 613, A12

Martayan, C., Frémat, Y., Hubert, A.-M., et al. 2007, A\&A, 462, 683

Martin, R. G., Nixon, C. J., Pringle, J. E., \& Livio, M. 2019, New Astron., 70, 7

Mayer, A., Deschamps, R., \& Jorissen, A. 2016, A\&A, 587, A30

McAlister, H. A., ten Brummelaar, T. A., Gies, D. R., et al. 2005, ApJ, 628, 439

Meilland, A., Millour, F., Kanaan, S., et al. 2012, A\&A, 538, A110

Meurs, E. J. A., Piters, A. J. M., Pols, O. R., et al. 1992, A\&A, 265, L41

Miroshnichenko, A. S., Bjorkman, K. S., \& Krugov, V. D. 2002, PASP, 114 1226

Motch, C., Lopes de Oliveira, R., Smith, M. A., et al. 2015, ApJ, 806, 177

Mourard, D., Monnier, J. D., Meilland, A., et al. 2015, A\&A, 577, A51

Nazé, Y., \& Motch, C. 2018, A\&A, 619, A148

Nazé, Y., Rauw, G., \& Cazorla, C. 2017, A\&A, 602, L5

Neiner, C., de Batz, B., Cochard, F., et al. 2011, AJ, 142, 149

Nemravová, J., Harmanec, P., Koubský, P., et al. 2012, A\&A, 537, A59

Ochsenbein, F., Bauer, P., \& Marcout, J. 2000, A\&AS, 143, 23

Okazaki, A. T., \& Negueruela, I. 2001, A\&A, 377, 161

Oskinova, L. M., Clarke, D., \& Pollock, A. M. T. 2001, A\&A, 378, L21

Packet, W. 1981, A\&A, 102, 17

Panoglou, D., Faes, D. M., Carciofi, A. C., et al. 2018, MNRAS, 473, 3039

Parmar, A. N., Israel, G. L., Stella, L., \& White, N. E. 1993, A\&A, 275, 227

Peters, G. J., \& Gies, D. R. 2005, ASP Conf. Ser., 337, 294

Peters, G. J., Pewett, T. D., Gies, D. R., Touhami, Y. N., \& Grundstrom, E. D. 2013, ApJ, 765, 2

Peters, G. J., Wang, L., Gies, D. R., \& Grundstrom, E. D. 2016, ApJ, 828, 47
Petrovic, J., Langer, N., \& van der Hucht, K. A. 2005, A\&A, 435, 1013 Pittard, J. M., \& Dawson, B. 2018, MNRAS, 477, 5640

Pols, O. R. 1994, A\&A, 290, 119

Pols, O. R., \& Marinus, M. 1994, A\&A, 288, 475

Pols, O. R., Cote, J., Waters, L. B. F. M., \& Heise, J. 1991, A\&A, 241, 419

Postnov, K., Oskinova, L., \& Torrejón, J. M. 2017, MNRAS, 465, L119

Raguzova, N. V. 2001, A\&A, 367, 848

Rappaport, S., Podsiadlowski, P., \& Horev, I. 2009, ApJ, 698, 666

Reid, A. H. N., Bolton, C. T., Crowe, R. A., et al. 1993, ApJ, 417, 320

Reig, P. 2011, Ap\&SS, 332, 1

Renzo, M., Zapartas, E., de Mink, S. E., et al. 2019, A\&A, 624, A66

Ricker, G. R., Vanderspek, R., Winn, J., et al. 2016, Proc. SPIE, 9904, 99042B

Rímulo, L. R., Carciofi, A. C., Vieira, R. G., et al. 2018, MNRAS, 476, 3555

Rivinius, T., Baade, D., Stefl, S., et al. 1998, ASP Conf. Ser., 135, 343

Rivinius, T., Štefl, S., \& Baade, D. 1999, A\&A, 348, 831

Rivinius, T., Baade, D., \& Stefl, S. 2003, A\&A, 411, 229

Rivinius, T., Štefl, S., Maintz, M., Stahl, O., \& Baade, D. 2004, A\&A, 427, 307

Rivinius, T., Carciofi, A. C., \& Martayan, C. 2013, A\&ARv, 21, 69

Rivinius, T., Baade, D., \& Carciofi, A. C. 2016, A\&A, 593, A106

Robinson, R. D., Smith, M. A., \& Henry, G. W. 2002, ApJ, 575, 435

Samus', N. N., Kazarovets, E. V., Durlevich, O. V., Kireeva, N. N., \& Pastukhova, E. N. 2017, Astron. Rep., 61, 80

Sano, T., Miyama, S. M., Umebayashi, T., \& Nakano, T. 2000, ApJ, 543, 486

Secchi, A. 1866, Astron. Nachr., 68, 63

Semaan, T., Hubert, A. M., Zorec, J., et al. 2018, A\&A, 613, A70

Shrader, C. R., Hamaguchi, K., Sturner, S. J., et al. 2015, ApJ, 799, 84

Smith, M. A. 2006, A\&A, 459, 215

Smith, M. A. 2019, PASP, 131, 044201

Smith, M. A., \& Balona, L. 2006, ApJ, 640, 491

Smith, M. A., Robinson, R. D., \& Hatzes, A. P. 1998, ApJ, 507, 945

Smith, M. A., Lopes de Oliveira, R., Motch, C., et al. 2012a, A\&A, 540, A53

Smith, M. A., Lopes de Oliveira, R., \& Motch, C. 2012b, ApJ, 755, 64

Smith, M. A., Lopes de Oliveira, R., \& Motch, C. 2016a, Adv. Space Res., 58, 782

Smith, M. A., Lopes de Oliveira, R., \& Motch, C. 2016b, ASP Conf. Ser., 506, 215

Smith, M. A., Lopes de Oliveira, R., \& Motch, C. 2017, MNRAS, 469, 1502

Soszynski, I., Udalski, A., Kubiak, M., et al. 2005, Acta Astron., 55, 331

Stee, P., Meilland, A., Bendjoya, P., et al. 2013, A\&A, 550, A65

Struve, O. 1931, ApJ, 73, 94

Struve, O. 1963, PASP, 75, 207

Tauris, T. M., \& van den Heuvel, E. P. J. 2006, Formation and Evolution of Compact Stellar X-ray Sources, 39, 623

Thaller, M. L., Bagnuolo, Jr., W. G., Gies, D. R., \& Penny, L. R. 1995, ApJ, 448, 878

Tsujimoto, M., Morihana, K., Hayashi, T., \& Kitaguchi, T. 2018, PASJ, 70, 109

Tycner, C., Gilbreath, G. C., Zavala, R. T., et al. 2006, AJ, 131, 2710

ud-Doula, A., Owocki, S. P., \& Kee, N. D. 2018, MNRAS, 478, 3049

Vanbeveren, D., Mennekens, N., Shara, M. M., \& Moffat, A. F. J. 2018, A\&A, 615, A65

Vink, J. S. 2017, A\&A, 607, L8

von Zeipel, H. 1924, MNRAS, 84, 665

Wade, G. A., Petit, V., Grunhut, J. H., Neiner, C., \& MiMeS Collaboration 2016, ASP Conf. Ser., 506, 207

Walker, G. A. H., Kuschnig, R., Matthews, J. M., et al. 2005, ApJ, 623, L145

Wang, L., Gies, D. R., \& Peters, G. J. 2017, ApJ, 843, 60

Wang, L., Gies, D. R., \& Peters, G. J. 2018, ApJ, 853, 156

Waters, L. B. F. M., Pols, O. R., Hogeveen, S. J., Cote, J., \& van den Heuvel, E. P. J. 1989, A\&A, 220, L1

Wellstein, S., \& Langer, N. 1999, A\&A, 350, 148

Wellstein, S., Langer, N., \& Braun, H. 2001, A\&A, 369, 939

Wenger, M., Ochsenbein, F., Egret, D., et al. 2000, A\&AS, 143, 9

Wisniewski, J. P., Draper, Z. H., Bjorkman, K. S., et al. 2010, ApJ, 709, 1306

Woosley, S. E. 2019, ApJ, 878, 49

Wright, E. L., Eisenhardt, P. R. M., Mainzer, A. K., et al. 2010, AJ, 140, 1868

Yang, S., Ninkov, Z., \& Walker, G. A. H. 1988, PASP, 100, 233

Yudin, R. V. 2001, A\&A, 368, 912

Zharikov, S. V., Miroshnichenko, A. S., Pollmann, E., et al. 2013, A\&A, 560, A30

Zorec, J., \& Briot, D. 1997, A\&A, 318, 443 